\title{
Detailed Re-derivation of Keplerian Orbit and Kaula's Satellite Orbit Perturbation Theory
}

\author{
Sung-Ho $\mathrm{Na}^{1 *}$, Tae-Seok $\mathrm{Bae}^{2}$, Jung Hyun $\mathrm{Jo}^{1}$, and Jong-Uk Park ${ }^{1}$ \\ ${ }^{1}$ Space Science Division, Korea Astronomy and Space Science Institute, \\ 776 Daedeokdae-ro, Hwaam-dong, Yuseong-gu, Daejeon, 305-348, Korea \\ ${ }^{2}$ Department of Geoinformation Engineering, Sejong University, \\ 98 Gunja-dong, Gwangjin-gu, Seoul, 143-747, Korea
}

\section{케플러궤도운동과 카울라의 인공위성궤도 섭동이론의 상세한 재유도

\author{
나성호 ${ }^{* *}$. 배태석 ${ }^{2} \cdot$ 조중현 ${ }^{\prime}$ 박종욱 ${ }^{1}$ \\ 1한국천문연구원 우주과학연구부, 305-348, 대전광역시 유성구 화암동 대덕대로 776 \\ ${ }^{2}$ 세종대학교 지구정보공학과, 143-747, 서울특별시 광진구 군자동 98
}

\begin{abstract}
Kaula's theory of satellite orbit and Kepler's law are re-visited. All the mathematical steps of derivation are thoroughly shown including the ones, which had been omitted in Kaula's original publication. Particularly in evaluations of the 15 independent Lagrange brackets, simplicity and clarity are attained by using orthogonal property of transformation matrix. Explanations of important physical concepts are included as well in the midway of derivation. One conceptual blunder of Kaula's is corrected.
\end{abstract}

Keywords: satellite orbit, Keplerian elements, Lagrange bracket

요 약: 카울라에 의해 완성되었던 인공위성궤도이론과 케플러의 법칙을 다시 다루었다. 카울라의 원문에서는 생략되었던 부분을 포함한 모든 수식유도과정을 상세히 기술하였다. 특히 15 개의 독립적인 라그랑지 괄호들을 계산하는 데에 변환행 렬의 직교성을 사용하여, 간결성과 명확성을 이루었다. 여러 중간단계에서 중요한 물리적인 개념들에 대한 설명도 추가하 였다. 카울라의 개념적 오류 한 개를 정정하였다.

주요어: 위성궤도, 케플러 요소, 라그랑지 괄호

\section{Introduction}

Since the first artificial satellite in 1957 , more than five thousand satellites were launched and have been used for various purposes including telecommunication, Earth observation, and positioning. Perhaps the most successful ones are NAVSTAR satellites composing Global Positioning System, which is widely used over the world.

Motion of satellite can be roughly approximated as a central force problem. And shape of its approximate

*Corresponding author: sunghona@kasi.re.kr Tel: 82-42-865-2062

Fax: 82-42-861-5610 orbit is an ellipse, that is determined by its energy and angular momentum. Planetary motions around the sun are also the same kind of elliptical orbits, and Kepler summarized their characteristics into his famous three laws. The great triumph of Newton was to deduce his universal law of gravitation from investigation of Keplerian orbit. Further enhancement of planetary orbital theory needs consideration of other nearby planets, and can be achieved by perturbation scheme. Satellite orbits are incessantly altered by several causes. Often numerical integrations are preferred to acquire precise orbit information for both planets and satellites. Set of Keplerian elements are relevant to represent planetary and satellite orbits (Table 1), and further minute deviations in the orbits can be 
Table 1. Six Keplerian elements and their symbols

\begin{tabular}{lc}
\hline \multicolumn{1}{c}{ Keplerian element } & Symbol \\
\hline semi-major axis & $a$ \\
eccentricity & $\varepsilon$ \\
inclination & $i$ \\
right ascension of ascending node & $\Omega$ \\
argument of perigee & $\omega$ \\
mean anomaly & $M$ \\
\hline
\end{tabular}

described by changes in the Keplerian elements.

The size and shape of an ellipse are determined by its semi-major axis and eccentricity, while the orientation of orbital plane is determined by the three angles - inclination, right ascension, and argument of perigee. Anomaly angle specifies the position of satellite on the ellipse. Satellite orbital geometry is illustrated with Keplerian elements in Fig. 1 and Fig. 2. If the Earth was of complete spherical symmetry, Earth's gravity would not cause any changes in the satellites orbital geometry. But the deviation of the Earth's gravity field from a purely central field causes slow but incessant changes in the satellite orbits. The dominant one of these changes is the westward/eastward drift of satellite orbits due to Earth's oblateness. Also there exist other causes of satellite orbit changes, such as gravitational pull by the Sun and the Moon, solar radiation pressure, and atmospheric drag, etc.

Kaula deduced the time rates of change in satellite's Keplerian elements as combinations of partial derivatives of the non-central potential in terms of Keplerian elements by using Lagrange brackets (Kaula, 1966). There were comparable studies prior to Kaula's work (Brouwer and Clemence, 1961, and Danby, 1962), but Kaula's formulation based on the choice of six variable including mean anomaly led lucidity and usefulness. Therefore his work has been regarded as standard treatment in the satellite orbit theory. Although his descriptions were accurate and almost immaculate, many of intermediate steps were omitted, for example, derivations for the Lagrange brackets and partial derivatives $\frac{\partial x_{i}}{\partial q_{k}}$ or $\frac{\partial \dot{x}_{i}}{\partial q_{k}}$ were not explicitly shown. In this article, we revisit Kepler's laws and Kaula's satellite orbit theory, and renew them with full

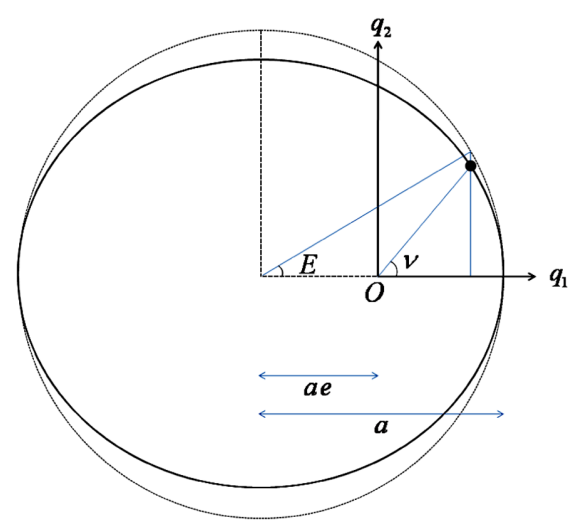

Fig. 1. Orbital size and shape of satellite motion: semimajor axis $a$, and eccentricity $\varepsilon$ are defined as shown in the figure. Focus is apart from the center by $a \varepsilon$. The origin of coordinate system $\left(q_{1}, q_{2}, q_{3}\right)$ is $O$. It is the center of mass of the two bodies, and coincide with the center of the Earth in practice. True anomaly $v$ refers to the position angle of satellite from perigee. Planetary motion can be described similarly.

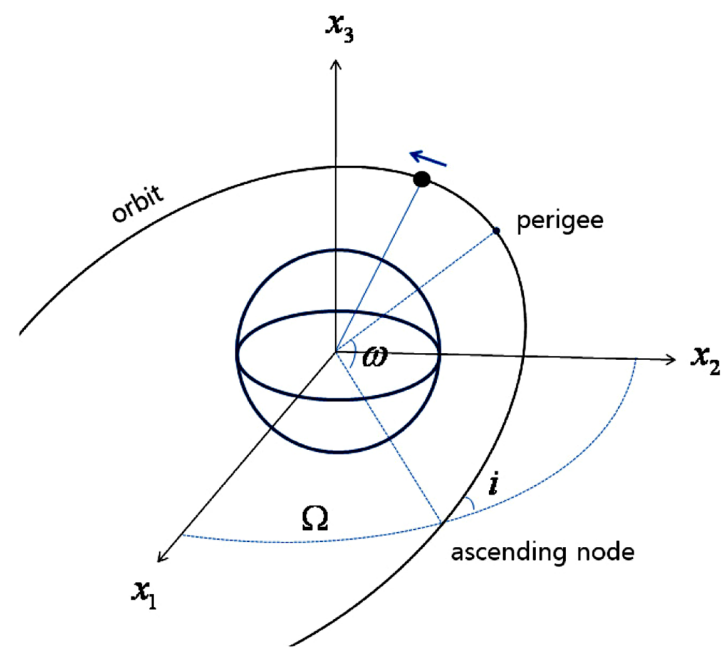

Fig. 2. Orientation of the satellite orbital plane: $x_{1}$ axis is toward the vernal equinox and $x_{3}$ axis is toward the north pole (conventional international origin). Inclination $i$ is the angle between the equatorial plane and the orbital plane. Right ascension $\Omega$ is the angle between the ascending node and $x_{1}$ axis. Argument of perigee $\omega$ is the angle between the ascending node and the perigee. Planetary motion can be described similarly in the heliocentric coordinate system.

description of each derivation steps. Unlike Kaula, for evaluations of Lagrange brackets, direct and lengthy algebra is avoided, but the orthogonal property of transformation matrix is utilized. 


\section{Keplerian Motion}

By analyzing the observations of planetary motions by Tycho Brahe (1546-1601), Johannes Kepler (15711630) found his famous three laws as follows: (1) planetary orbits are ellipses having the sun on the focus, (2) each radius vector to a planet sweeps same area during any given same time interval, and (3) the square of each orbital period is proportional to the cube of each orbital semi-major axis. Issac Newton (1642-1727) found that three Kepler's laws could be derived from one fundamental law - 'the universal law of gravitation.' And his inverse square force law for gravity is still in use at various astronomical, astronautical and geophysical studies, even though Newton's law was surpassed by more fundamental law of general relativity after Albert Einstein (18791955). Kepler laws are treated as one of standard materials in classical/celestial mechanics, and a brief but self consistent description is given here.

Newton's law of gravitation is expressed as

$$
\vec{f}_{12}=-G_{\mid \overrightarrow{r_{1}} m_{2}} \underset{\left|\overrightarrow{r_{1}}-\vec{r}_{2}\right|^{2}}{\vec{r}}
$$

where $\vec{f}_{12}$ is the gravitational force acting on particle 1 by particle $2, G$ is the constant of gravitation, $m_{1}$ and $m_{2}$ are the particle masses, $\vec{r}_{1}$ and $\vec{r}_{2}$ are positions, and $\hat{r}$ is the unit vector of $\vec{r}_{1}-\vec{r}_{2}$, i.e. $\hat{r}=\frac{\vec{r}_{1}-\vec{r}_{2}}{\left|\overrightarrow{r_{1}-\vec{r}_{2}}\right|}$.

As for conservative force like gravity, which can be expressed as gradient of a potential, it is more convenient to use the potential in most formulations rather than the force itself. The equations of motion for two bodies forcing each other through a gravity potential $V\left(\left|\vec{r}_{1}-\vec{r}_{2}\right|\right)=V(r)$ can be written as follows.

$$
\stackrel{\vec{F}}{m_{1} r_{1}}=-\vec{\nabla}_{1} V\left(\left|\overrightarrow{r_{1}}-\vec{r}_{2}\right|\right)=-\stackrel{\overrightarrow{\vec{r}}}{m_{2}}=+\vec{\nabla}_{2} V\left(\left|\overrightarrow{r_{1}}-\overrightarrow{r_{2}}\right|\right),
$$

where $\vec{\nabla}_{i}$ refers to 'gradient operation with respect to position $\vec{r}_{i}$. We take the sign convention for the potential as expressed by Eq. (2-2) to maintain the equation of mechanical energy conservation as its original definition. If we rewrite the equation of motion in terms of $\vec{R}$, which is the center of mass, and $\vec{r}=\vec{r}_{1}-\vec{r}_{2}$, then we attain alternative expression as follows.

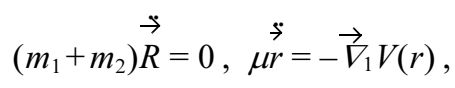

where reduced mass is defined as $\mu=\frac{m_{1} m_{2}}{m_{1}+m_{2}}$. Then the problem of two bodies under gravitational attraction is converted to one body central force problem.

$$
\stackrel{\vec{r}}{\mu r}=-G m_{1} m_{2} \frac{\vec{r}}{r^{3}} \Rightarrow \stackrel{\vec{r}}{r}=-G\left(m_{1}+m_{2}\right) \frac{\vec{r}}{r^{3}}
$$

Polar coordinates $r$ and $\phi$ are the relevant coordinates for central force problem. Polar coordinates and cartesian coordinates are related through the following relation.

$$
x=r \cos \phi, y=r \sin \phi
$$

Since no torque is exerted by central force, conservation of angular momentum evidently holds.

$$
\begin{aligned}
& l=L_{z}=(\vec{r} \times \vec{p})_{z}=x p_{y}-y p_{x}=\mu(x \dot{y}-y \dot{x})=\mu r^{2} \dot{\phi} \\
& =\text { constant }
\end{aligned}
$$

The 2nd law of Kepler is attained accordingly.

$$
d A=\frac{1}{2} r(r d \phi) \Rightarrow \frac{d A}{d t}=\frac{1}{2} r^{2} \dot{\phi}=\frac{l}{2 \mu}=\text { constant }
$$

Another constant of central force motion is the total energy $F$, which is sum of the kinetic energy $T$ and the potential energy $V$ (We reserve the symbol $E$ to represent the eccentric anomaly).

$$
\begin{aligned}
F=T+V & =\frac{1}{2} \mu\left(\dot{r}^{2}+r^{2} \dot{\phi}^{2}\right)-G \frac{m_{1} m_{2}}{r} \\
& =\frac{1}{2} \mu\left(\dot{r}^{2}+r^{2} \dot{\phi}^{2}\right)-\gamma \mu r^{-1},
\end{aligned}
$$

where $\gamma$ is defined as $\gamma=G\left(m_{1}+m_{2}\right)$. For planets in the solar system, $\mu$ is close to the mass of each planet, but deviates in the same order of 'mass ratio' between the planet and the sun. For a satellite in the 
Earth's gravity field, $\mu$ is given as the mass of the satellite itself (practically negligible difference).

It is convenient to use effective potential $V_{\text {eff }}$, which is defined as

$$
V_{\text {eff }}(r)=-\gamma \mu r^{-1}+\frac{1}{2} \mu r^{2} \dot{\phi}^{2}=-\gamma \mu r^{-1}+\frac{l^{2}}{2 \mu r^{2}}
$$

Then we have useful expression for time rate of radius from Eq. (2-8) as follows.

$$
\frac{d r}{d t}= \pm \sqrt{\frac{2}{\mu}\left(F-V_{e f f}\right)}
$$

And from the definition of angular momentum, $l=$ $\mu r^{2} \frac{d \phi}{d t}$, we have the following relations.

$$
\begin{aligned}
& d \phi=\frac{l}{\mu r^{2}} d t=\frac{l}{\mu r^{2}} \times \frac{ \pm d r}{\sqrt{\frac{2}{\mu}\left(F-V_{e f f}\right)}} \\
& \phi-\phi_{1}= \pm \frac{l}{\sqrt{2 \mu}} \int \frac{d r}{r^{2} \sqrt{F-V_{e f f}}}
\end{aligned}
$$

With a new variable $u=\frac{1}{r}$, Eq. (2-12) can be rewritten as

$$
\phi-\phi_{1}= \pm \frac{l}{\sqrt{2 \mu}} \int \frac{d r}{r^{2} \sqrt{F-V_{e f f}}}=\mp \int \frac{d u}{\sqrt{\frac{2 \mu F}{l^{2}}+\frac{2 \mu^{2} \gamma u}{l^{2}}-u^{2}}}
$$

Integration of Eq. (2-13) leads to the following (Gradshteyn and Ryzhik, 1965).

$$
v=\phi-\phi_{0}=\phi-\left(\phi_{1}-\frac{\pi}{2}\right)=\arccos \left[\frac{u l^{2} / \mu^{2} \gamma-1}{\sqrt{1+2 F l^{2} / \mu^{3} \gamma^{2}}}\right]
$$

Rearranging terms, we find one of conic sections.

$$
\frac{1}{r}=C\left[1+\varepsilon \cos \left(\phi-\phi_{0}\right)\right]
$$

where $C$ and $\varepsilon$ are defined as

$$
C=\frac{\mu^{2} \gamma}{l^{2}}, \quad \varepsilon=\sqrt{1+\frac{2 F l^{2}}{\mu^{3} \gamma^{2}}}
$$

So the Kepler's 1st law is verified. For Keplerian motion, the eccentricity $\varepsilon$ has its value between 0 and 1. In alternative expression, Eq. (2-15a) can be rewritten as follows.

$$
\frac{x^{\prime 2}}{a^{2}}+\frac{y^{\prime 2}}{b^{2}}=1
$$

where we define $a$ and $b$ as

$$
a=\frac{1}{C\left(1-\varepsilon^{2}\right)}, b=a \sqrt{1-\varepsilon^{2}}
$$

Using Eq. (2-6) and total area of ellipse $A$, we find the period of Keplerian orbital motion as follows.

$$
\tau=\frac{A}{d A / d t}=2 \pi \sqrt{\frac{a^{3}}{\gamma}}
$$

where $A$ is given as

$$
A=\pi a b=\pi a^{2} \sqrt{1-\varepsilon^{2}}=\pi a^{2} \sqrt{\frac{2|F| l^{2}}{\mu^{3} \gamma^{2}}}=\frac{\pi l}{\mu} \sqrt{\frac{a^{3}}{\gamma}}
$$

In fact, Kepler's 3rd law is an approximate expression of Eq. (2-17).

We hereby add a useful relation among true anomaly $v$, eccentric anomaly $E$, and mean anomaly $M$. By differentiating Eq. (2-15), we have

$$
\frac{d r}{d v}=-r^{2} C \varepsilon \sin v
$$

Using the definition of $C$ in Eq. (2-16b), we find following relation

$$
\frac{d r}{d v}=\frac{d(1 / r)}{d v}\left(-r^{2}\right)=-r^{2} C \varepsilon \sin v=\frac{\varepsilon r^{2}}{a\left(1-\varepsilon^{2}\right)} \sin v
$$

We can write the following relations (Fig. 1).

$$
\begin{aligned}
& q_{1}=x^{\prime}-a \varepsilon=a \cos E-a \varepsilon, \\
& q_{2}=y^{\prime}=b \sin E=a \sqrt{1-\varepsilon^{2}} \sin E
\end{aligned}
$$

Accordingly we have

$$
r=\sqrt{q_{1}^{2}+q_{2}^{2}}=a(1-\varepsilon \cos E)
$$


Differentiating Eq. (2-20), we have

$$
\frac{d r}{d E}=a \varepsilon \sin E
$$

From Eqs. (2-18b) and (2-21), we find the following relation.

$$
\frac{\varepsilon r^{2}}{a\left(1-\varepsilon^{2}\right)} \sin v d v=a \varepsilon \sin E d E
$$

After replacement of $d v$ by using the definition of angular momentum as $d v=\frac{1}{\mu r^{2}} d t$ and use of another equality $r \sin v=q_{2}$, we find the following.

$$
d t=\frac{a^{2} \mu \sqrt{1-\varepsilon^{2}}}{l}(1-\varepsilon \cos E) d E=\frac{1-\varepsilon \cos E}{n} d E
$$

which leads to

$$
t-t_{0}=\frac{a^{2} \mu \sqrt{1-\varepsilon^{2}}}{l}(E-\varepsilon \sin E)=\frac{1}{n}(E-\varepsilon \sin E),
$$

where $t_{0}$ is perigee passing epoch and mean angular velocity $n$ is defined as

$$
n=\frac{l}{a^{2} \mu \sqrt{1-\varepsilon^{2}}}=\sqrt{\frac{\gamma}{a^{3}}}=\frac{2 \pi}{\tau} .
$$

After defining mean anomaly as

$$
M=E-\varepsilon \sin E,
$$

we can simply rewrite Eq. (2-22a) as

$$
n\left(t-t_{0}\right)=M
$$

To calculate the position on the orbital ellipse, we first calculate mean anomaly for given epoch using Eq. (2-23b). Next we acquire eccentric anomaly through iterations or series expression based on the relation (2-23a). With known E, Eqs. (2-19) yield the position. Components of orbital velocity are expressed as time derivatives of position coordinates.

$$
\dot{q}_{1}=-a \sin E \frac{d E}{d t}, \quad \dot{q}_{2}=a \sqrt{1-\varepsilon^{2}} \cos E \frac{d E}{d t}
$$

, where $\frac{d E}{d t}$ is readily acquired from Eq. (2-22a) as

$$
\frac{d E}{d t}=\frac{n}{1-\varepsilon \cos E}
$$

Transformation from $q$-coordinates in the orbital plane to the inertial coordinate $\left(x_{1}, x_{2}, x_{3}\right)$ can be done by multiplying three rotation matrices in series.

$$
\left(\begin{array}{l}
x_{1} \\
x_{2} \\
x_{3}
\end{array}\right)=R_{3}(-\Omega) R_{1}(-i) R_{3}(-\omega)\left(\begin{array}{c}
q_{1} \\
q_{2} \\
0
\end{array}\right),
$$

where $R_{3}(\theta)=\left(\begin{array}{ccc}\cos \theta & \sin \theta & 0 \\ -\sin \theta & \cos \theta & 0 \\ 0 & 0 & 1\end{array}\right)$ and

$R_{1}(\theta)=\left(\begin{array}{ccc}1 & 0 & 0 \\ 0 & \cos \theta & \sin \theta \\ 0-\sin \theta & \cos \theta\end{array}\right)$.

Components of velocity are transformed in the same way as Eq. (2-25).

A few supplementary formulae are given below. Although these relations were not needed in the former derivations up to Eq. (2-24), they have fundamental meaning and convenient usage. We may equate kinetic energy directly from its definition as $T=\frac{1}{2} \mu v^{2}=\frac{1}{2} \mu\left(\dot{q}_{1}^{2}+\dot{q}_{2}^{2}\right)$. And the kinetic energy compose part of total energy with potential energy $V$.

$$
\begin{aligned}
& v^{2}=\left(\dot{q}_{1}^{2}+\dot{q}_{2}^{2}\right) \Rightarrow v^{2}=\chi\left(\frac{2}{r}-\frac{1}{a}\right) \\
& T+V=\frac{1}{2} \mu v^{2}-\frac{\gamma \mu}{r}=-\frac{\gamma \mu}{2 a}=\text { total Energy }
\end{aligned}
$$

And, from Eq. (2-19), we find a relation between true anomaly $v$ and eccentric anomaly $E$.

$$
\tan v=\frac{\sqrt{1-\varepsilon^{2}} \sin E}{\cos E-\varepsilon}
$$

There are other methods to deduce the Kepler's law from the Newton's law of gravitation than the one of this section. Two of them are given in Appendix. 


\section{Equation of satellite motion under non-central potential: Kaula's theory}

Kaula gave expressions for the time rate of Keplerian elements of satellite orbit in non-central gravitational field. His formulation is extremely neat and almost free from defects (containing only one minor conceptual mistake and one typographical error). In this section, we faithfully follow his derivations with one minor correction and the steps which had been omitted in Kaula's (Kaula, 1966). Our notations are same as the ones of Kaula except the potential energy and the total energy.

Newtonian equation of motion for satellite is given as $\overrightarrow{m r}=-\vec{\nabla}_{1} V(r)$. From now on, we take the mass of satellite $m$ as unity and the potential defined accordingly. Then the $i$-th component of equation of motion becomes

$$
\frac{d}{d t}\left(m \dot{x}_{i}\right) \Rightarrow \frac{d \dot{x}_{i}}{d t}=-\frac{\partial V}{\partial x_{i}}
$$

We want to represent the equation of motion in terms of Keplerian orbital elements $s_{k}: a, \varepsilon, i, \omega, \Omega, M$. Also we want to know the time derivatives of Keplerian orbital elements. To this end, we first rewrite time derivative of position as $\dot{x}_{i}=\frac{d x_{i}}{d t}=\sum_{k=1}^{6} \frac{\partial x_{i}}{\partial s_{k}} \frac{d s_{k}}{d t}$. Similarly we rewrite $\frac{d \dot{x}_{i}}{d t}=\sum_{k=1}^{6} \frac{\partial \dot{x}_{i}}{\partial s_{k}} \frac{d s_{k}}{d t}$ and $\frac{\partial V}{\partial x_{i}}=\sum_{k=1}^{6} \frac{\partial V}{\partial s_{k}} \frac{\partial s_{k}}{\partial x_{i}}$.

Then Eq. (3-1) can be rewritten as the following.

$$
\frac{d \dot{x}_{i}}{d t}=\sum_{k=1}^{6} \frac{\partial \dot{x}_{i}}{\partial s_{k}} \frac{d s_{k}}{d t}=-\frac{\partial V}{\partial x_{i}}=-\sum_{k=1}^{6} \frac{\partial V}{\partial s_{k}} \frac{\partial s_{k}}{\partial x_{i}}
$$

Now multiply $-\frac{\partial \dot{x}_{i}}{\partial s_{l}}$ to Eq. (3-2) and sum it over index $i$, then we have

$$
-\sum_{i=1}^{3} \frac{\partial \dot{x}_{i}}{\partial s_{l}} \sum_{k=1}^{6} \frac{\partial x_{i}}{\partial s_{k}} \frac{d s_{k}}{d t}=-\sum_{i=1}^{3} \frac{\partial \dot{x}_{i}}{\partial s_{l}} \dot{x}_{i}
$$

Also multiplying $\frac{\partial x_{i}}{\partial s_{l}}$ to Eq. (3-2) and summing them over index $i$, then we have

$$
\sum_{i=1}^{3} \frac{\partial x_{i}}{\partial s_{l}} \sum_{k=1}^{6} \frac{\partial \dot{x}_{i}}{\partial s_{k}} \frac{d s_{k}}{d t}=-\sum_{i=1}^{3} \frac{\partial x_{i}}{\partial s_{l}} \frac{\partial V}{\partial x_{i}}
$$

By adding Eqs. (3-3a) and (3-3b) we have

$$
\sum_{k=1}^{6} \sum_{i=1}^{3}\left(\frac{\partial x_{i}}{\partial s_{l}} \frac{\partial \dot{x}_{i}}{\partial s_{k}}-\frac{\partial \dot{x}_{i}}{\partial s_{l}} \frac{\partial x_{i}}{\partial s_{k}}\right) \frac{d s_{k}}{d t}=-\sum_{i=1}^{3} \frac{\partial \dot{x}_{i}}{\partial s_{l}} \dot{x}_{i}-\sum_{i=1}^{3} \frac{\partial x_{i}}{\partial s_{l}} \frac{\partial V}{\partial x_{i}} .
$$

The left hand side of Eq. (3-3) can be rewritten as $\sum_{k=1}^{6}\left[s_{l}, s_{k}\right] \frac{d s_{k}}{d t}$, where Lagrange bracket $\left[s_{l}, s_{k}\right]$ is defined as

$$
\left[s_{l}, s_{k}\right]=\sum_{i=1}^{3}\left(\frac{\partial x_{i}}{\partial s_{l}} \frac{\partial \dot{x}_{i}}{\partial s_{k}}-\frac{\partial \dot{x}_{i}}{\partial s_{l}} \frac{\partial x_{i}}{\partial s_{k}}\right)
$$

Also the right hand side of Eq. (3-3) can be rewritten as 


$$
-\sum_{i=1}^{3} \frac{\partial \dot{x}_{i}}{\partial s_{l}} \dot{x}_{i}-\sum_{i=1}^{3} \frac{\partial x_{i}}{\partial s_{l}} \frac{\partial V}{\partial x_{i}}=-\frac{1}{2} \frac{\partial}{\partial s_{l}}\left(\dot{x}_{i}^{2}\right)-\frac{\partial V}{\partial s_{l}}=-\frac{\partial}{\partial s_{l}}\left(\frac{1}{2} \dot{x}_{i}^{2}+V\right)=\frac{\partial}{\partial s_{l}}(\text { total Energy })=-\frac{\partial F}{\partial s_{l}},
$$

where $F$ is defined as the total energy as $F=T+V$. So Eq. (3-3) is rewritten as

$$
\sum_{k=1}^{6}\left[s_{l}, s_{k}\right] \frac{d s_{k}}{d t}=-\frac{\partial F}{\partial s_{l}}
$$

The extraordinary usefulness of Lagrange bracket is its time invariance. Lagrange bracket and Poisson bracket are two sets of canonical invariant, i.e. constant under canonical transformation, and they compose an inverse relation. In fact Sterne derived the equivalent formulae for satellite motion by using Poisson bracket (Sterne, 1960), and his derivation is briefly described in Appendix. The time invariance of Lagrange bracket can be explicitly shown as follows.

Consider time derivative of Lagrange bracket as

$$
\frac{\partial}{\partial t}\left[s_{l}, s_{k}\right]=\sum_{i=1}^{3}\left(\frac{\partial^{2} x_{i}}{\partial t \partial s_{l}} \frac{\partial \dot{x}_{i}}{\partial s_{k}}+\frac{\partial x_{i}}{\partial s_{l}} \frac{\partial^{2} \dot{x}_{i}}{\partial t \partial s_{k}}-\frac{\partial^{2} \dot{x}_{i}}{\partial t \partial s_{l}} \frac{\partial x_{i}}{\partial s_{k}}+\frac{\partial \dot{x}_{i}}{\partial s_{l}} \frac{\partial^{2} x_{i}}{\partial t \partial s_{k}}\right)
$$

By manipulation on the right hand side of this relation - adding and deducting two terms $\frac{\partial x_{i}}{\partial t} \frac{\partial^{2} \dot{x}_{i}}{\partial s_{l} \partial s_{k}}$ and $-\frac{\partial^{2} x_{i}}{\partial s_{l} \partial s_{k}} \frac{\partial \dot{x}_{i}}{\partial t}$ etc, we have the time derivative of Lagrange bracket as follows.

$$
\begin{aligned}
\frac{\partial}{\partial t}\left[s_{l}, s_{k}\right] & =\frac{\partial}{\partial s_{l}} \sum_{i=1}^{3}\left(\frac{\partial x_{i}}{\partial t} \frac{\partial \dot{x}_{i}}{\partial s_{k}}-\frac{\partial x_{i}}{\partial s_{k}} \frac{\partial \dot{x}_{i}}{\partial t}\right)-\frac{\partial}{\partial s_{k}} \sum_{i=1}^{3}\left(\frac{\partial x_{i}}{\partial t} \frac{\partial \dot{x}_{i}}{\partial s_{l}}-\frac{\partial x_{i}}{\partial s_{l}} \frac{\partial \dot{x}_{i}}{\partial t}\right) \\
& =\frac{\partial}{\partial s_{l}} \sum_{i=1}^{3}\left(\dot{x}_{i} \frac{\partial \dot{x}_{i}}{\partial s_{k}}-\frac{\partial x_{i}}{\partial s_{k}} \ddot{x}_{i}\right)-\frac{\partial}{\partial s_{k_{i}}} \sum_{i=1}^{3}\left(\dot{x}_{i} \frac{\partial \dot{x}_{i}}{\partial s_{l}}-\frac{\partial x_{i}}{\partial s_{l}} \ddot{x}_{i}\right) \\
& =\frac{\partial}{\partial s_{l}} \sum_{i=1}^{3}\left(\dot{x}_{i} \frac{\partial \dot{x}_{i}}{\partial s_{k}}+\frac{\partial x_{i}}{\partial s_{k}} \frac{\partial V}{\partial x_{l}}\right)-\frac{\partial}{\partial s_{k}} \sum_{i=1}^{3}\left(\dot{x}_{i} \frac{\partial \dot{x}_{i}}{\partial s_{l}}+\frac{\partial x_{i}}{\partial s_{l}} \frac{\partial V}{\partial x_{i}}\right) \\
& =\frac{\partial}{\partial s_{l}} \frac{\partial(T+V)}{\partial s_{k}}-\frac{\partial}{\partial s_{k}} \frac{\partial(T+V)}{\partial s_{l}}=0,
\end{aligned}
$$

where two relations,

$$
\frac{\partial T}{\partial s_{k}}=\frac{\partial}{\partial s_{k}}\left(\sum_{i=1}^{3} \frac{1}{2} \dot{x}_{i}^{2}\right)=\sum_{i=1}^{3} \dot{x}_{i} \frac{\partial \dot{x}_{i}}{\partial s_{k}} \text { and } \ddot{x}_{i}=-\frac{\partial V}{\partial x_{i}}
$$

have been used in later step. Here, Kaula had made a blunder to specify $V$ as purely of degree 1 potential.

To solve Eq. (3-3) for the time derivatives of Keplerian orbital elements of satellite; $\frac{d s_{k}}{d t}$, we need to know the whole set of Lagrange brackets $\left[s_{l}, s_{k}\right]$. Total number of Lagrange brackets for set of six variables are 36, but the number of independent Lagrange brackets are 15 , because of their antisymmetry; $\left[s_{l}, s_{k}\right]=-\left[s_{k}, s_{l}\right]$. Since Lagrange brackets are time invariant, they can be evaluated at any convenient point, such as perigee. Followings are derivations of those 15 independent Lagrange brackets.

To evaluate Lagrange bracket $\left[s_{l}, s_{k}\right]=\sum_{i=1}^{3}\left(\frac{\partial x_{i}}{\partial s_{l}} \frac{\partial \dot{x}_{i}}{\partial s_{k}}-\frac{\partial \dot{x}_{i}}{\partial s_{l}} \frac{\partial x_{i}}{\partial s_{k}}\right)$, we need to know the partial derivatives of position and velocity with respect to the orbital elements $s_{k}$, i.e. $\frac{\partial x_{i}}{\partial s_{k}}$ and $\frac{\partial \dot{x}_{i}}{\partial s_{k}}$. Let us go back to Eqs. (2-24), (2-25), and their associates. 


$$
\begin{aligned}
& \left(\begin{array}{l}
x_{1} \\
x_{2} \\
x_{3}
\end{array}\right)=R_{3}(-\Omega) R_{1}(-i) R_{3}(-\omega)\left(\begin{array}{l}
q_{1} \\
q_{2} \\
q_{3}
\end{array}\right),\left(\begin{array}{l}
\dot{x}_{1} \\
\dot{x}_{2} \\
\dot{x}_{3}
\end{array}\right)=R_{3}(-\Omega) R_{1}(-i) R_{3}(-\omega)\left(\begin{array}{l}
\dot{q}_{1} \\
\dot{q}_{2} \\
\dot{q}_{3}
\end{array}\right) \\
& \left(\begin{array}{l}
q_{1} \\
q_{2} \\
q_{3}
\end{array}\right)=\left(\begin{array}{c}
a(\cos E-\varepsilon) \\
a \sqrt{1-\varepsilon^{2}} \sin E \\
0
\end{array}\right),\left(\begin{array}{l}
\dot{q}_{1} \\
\dot{q}_{2} \\
\dot{q}_{3}
\end{array}\right)=\frac{n a}{1-\varepsilon \cos E}\left(\begin{array}{c}
-\sin E \\
\sqrt{1-\varepsilon^{2}} \cos E \\
0
\end{array}\right)
\end{aligned}
$$

For brevity the transformation matrix $R_{3}(-\Omega) R_{1}(-i) R_{3}(-\omega)$ is replaced by $X(\Omega, i, \omega)$.

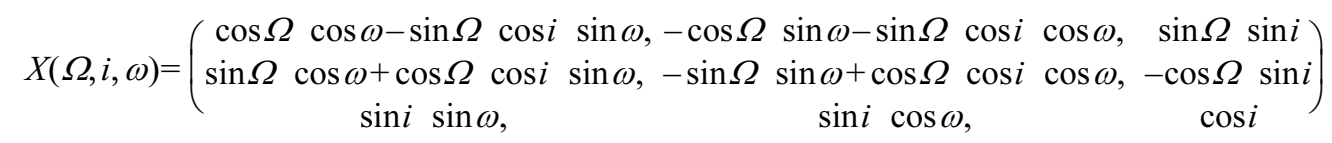

Then $x_{i}=\sum_{k=1}^{3} X_{i k} q_{k}$ and $\dot{x}_{i}=\sum_{k=1}^{3} X_{i k} \dot{q}_{k}$. Here we review a property of the transformation matrix $X(\Omega, i, \omega)=$ $R_{3}(-\Omega) R_{1}(-i) R_{3}(-\omega)$. Like other rotational transformation matrix, $X(\Omega, i, \omega)$ belongs to the group of orthogonal matrices. So the transpose of $X$ is its inverse, i.e. $X^{T}=X^{-1}$, or equivalently $X^{T} X=I$. In other words, the property $\sum_{k} X_{i k}^{T} X_{k j}=\sum_{k} X_{k i} X_{k j}=\delta_{i j}$ claims that the column vectors of are orthonormal to each other. Similarly the row vectors of $X$ are also orthonormal to each other.

$$
X X^{T}=I \quad \Rightarrow \quad \sum_{k} X_{i k} X_{k j}^{T}=\sum_{k} X_{i k} X_{j k}=\delta_{i j}
$$

Now coordinate transforms of position and velocity are rewritten as follows.

$$
\begin{aligned}
& \left(\begin{array}{l}
x_{1} \\
x_{2} \\
x_{3}
\end{array}\right)=X(\Omega, i, \omega)\left(\begin{array}{l}
q_{1} \\
q_{2} \\
q_{3}
\end{array}\right)=\left(\begin{array}{lll}
X_{11} & X_{12} & X_{13} \\
X_{21} & X_{22} & X_{23} \\
X_{31} & X_{32} & X_{33}
\end{array}\right)\left(\begin{array}{c}
a(\cos E-\varepsilon) \\
a \sqrt{1-\varepsilon^{2}} \sin E \\
0
\end{array}\right) \\
& \left(\begin{array}{l}
\dot{x}_{1} \\
\dot{x}_{2} \\
\dot{x}_{3}
\end{array}\right)=X(\Omega, i, \omega)\left(\begin{array}{l}
\dot{q}_{1} \\
\dot{q}_{2} \\
\dot{q}_{3}
\end{array}\right)=\left(\begin{array}{lll}
X_{11} & X_{12} & X_{13} \\
X_{21} & X_{22} & X_{23} \\
X_{31} & X_{32} & X_{33}
\end{array}\right) \frac{n a}{1-\varepsilon \cos E}\left(\begin{array}{c}
-\sin E \\
\sqrt{1-\varepsilon^{2}} \cos E \\
0
\end{array}\right)
\end{aligned}
$$

The partial derivatives $\frac{\partial x_{i}}{\partial \Omega}, \frac{\partial x_{i}}{\partial i}$, and $\frac{\partial x_{i}}{\partial \omega}$ at perigee are acquired accordingly as the followings.

$$
\begin{aligned}
\frac{\partial}{\partial \Omega}\left(\begin{array}{l}
x_{1} \\
x_{2} \\
x_{3}
\end{array}\right) & =\frac{\partial}{\partial \Omega}\left(\begin{array}{lll}
X_{11} & X_{12} & X_{13} \\
X_{21} & X_{22} & X_{23} \\
X_{31} & X_{32} & X_{33}
\end{array}\right)\left(\begin{array}{c}
a(1-\varepsilon) \\
0 \\
0
\end{array}\right)=\frac{\partial}{\partial \Omega}\left(\begin{array}{l}
X_{11} \\
X_{21} \\
X_{31}
\end{array}\right) a(1-\varepsilon) \\
& =\left(\begin{array}{ccc}
-\sin \Omega & \cos \omega-\cos \Omega \cos i \sin \omega \\
\cos \Omega & \cos \omega-\sin \Omega & \cos i \sin \omega \\
0
\end{array}\right) a(1-\varepsilon)=\left(\begin{array}{c}
-X_{21} \\
X_{11} \\
0
\end{array}\right) a(1-\varepsilon)
\end{aligned}
$$




$$
\begin{aligned}
& \frac{\partial}{\partial i}\left(\begin{array}{l}
x_{1} \\
x_{2} \\
x_{3}
\end{array}\right)=\frac{\partial}{\partial i}\left(\begin{array}{lll}
X_{11} & X_{12} & X_{13} \\
X_{21} & X_{22} & X_{23} \\
X_{31} & X_{32} & X_{33}
\end{array}\right)\left(\begin{array}{c}
a(1-\varepsilon) \\
0 \\
0
\end{array}\right)=\frac{\partial}{\partial i}\left(\begin{array}{l}
X_{11} \\
X_{21} \\
X_{31}
\end{array}\right) a(1-\varepsilon)
\end{aligned}
$$

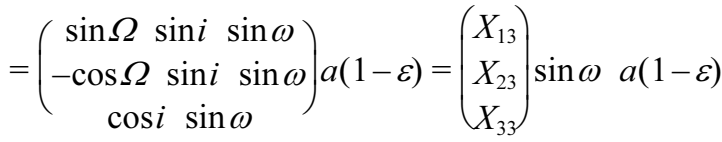

$$
\begin{aligned}
& \frac{\partial}{\partial \omega}\left(\begin{array}{l}
x_{1} \\
x_{2} \\
x_{3}
\end{array}\right)=\frac{\partial}{\partial \omega}\left(\begin{array}{lll}
X_{11} & X_{12} & X_{13} \\
X_{21} & X_{22} & X_{23} \\
X_{31} & X_{32} & X_{33}
\end{array}\right)\left(\begin{array}{c}
a(1-\varepsilon) \\
0 \\
0
\end{array}\right)=\frac{\partial}{\partial \omega}\left(\begin{array}{l}
X_{11} \\
X_{21} \\
X_{31}
\end{array}\right) a(1-\varepsilon)
\end{aligned}
$$

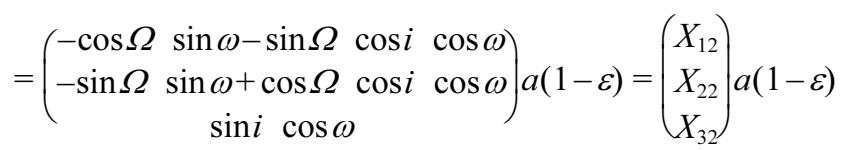

Evaluation of $\frac{\partial x_{i}}{\partial a}$ takes a little more attention, because $\frac{\partial E}{\partial a}$ is needed as follows.

$$
\frac{\partial}{\partial a}\left(\begin{array}{l}
x_{1} \\
x_{2} \\
x_{3}
\end{array}\right)=\left(\begin{array}{lll}
X_{11} & X_{12} & X_{13} \\
X_{21} & X_{22} & X_{23} \\
X_{31} & X_{32} & X_{33}
\end{array}\right) \frac{\partial}{\partial a}\left(\begin{array}{c}
q_{1} \\
q_{2} \\
0
\end{array}\right)
$$

We have

$$
\begin{aligned}
& \frac{\partial q_{1}}{\partial a}=\frac{\partial}{\partial a}[a(\cos E-\varepsilon)]=\cos E-\varepsilon-a \sin E \frac{\partial E}{\partial a}, \text { and } \\
& \frac{\partial q_{2}}{\partial a}=\frac{\partial}{\partial a}\left[a \sqrt{1-\varepsilon^{2}} \sin E\right]=a \sqrt{1-\varepsilon^{2}} \sin E+a \sqrt{1-\varepsilon^{2}} \cos E \frac{\partial E}{\partial a}
\end{aligned}
$$

To calculate $\frac{\partial E}{\partial a}$, we can use the relation

$$
E-\varepsilon \sin E=M=n\left(t-t_{0}\right)+M_{0},
$$

where $n=\sqrt{\frac{\gamma}{a^{3}}}$ (Eqs. (2-22) and (2-23)).

After differentiation on this relation with respect to semi-major axis $a$, we have

$$
\frac{\partial E}{\partial a}-\varepsilon \cos E \frac{\partial E}{\partial a}=\frac{\partial n}{\partial a}\left(t-t_{0}\right)=-\frac{3 n}{2 a}\left(t-t_{0}\right)=-\frac{3\left(M-M_{0}\right)}{2 a}=-\frac{3 M_{1}}{2 a},
$$

where $M_{1}$ is defined as $M_{1}=M\left(t_{1}\right)-M_{0}$ with perigee passing time $t_{1}$. Since $M_{1}$ is nothing but an arbitrary integral multiple of $2 \pi, M_{1}$ do not exist in expressions of Lagrange brackets or equation of motion for satellite. At perigee we have following equalities as

$$
\frac{\partial E}{\partial a}=\frac{-3 M_{1}}{2 a(1-\varepsilon)}, \frac{\partial q_{1}}{\partial a}=1-\varepsilon, \frac{\partial q_{2}}{\partial a}=a \sqrt{1-\varepsilon} \frac{\partial E}{\partial a}=-\frac{3}{2} \sqrt{\frac{1+\varepsilon}{1-\varepsilon}} M_{1}
$$

Then, at perigee, we have $\frac{\partial x_{i}}{\partial a}$ as follows. 


$$
\frac{\partial}{\partial a}\left(\begin{array}{l}
x_{1} \\
x_{2} \\
x_{3}
\end{array}\right)=\left(\begin{array}{lll}
X_{11} & X_{12} & X_{13} \\
X_{21} & X_{22} & X_{23} \\
X_{31} & X_{32} & X_{33}
\end{array}\right) \frac{\partial}{\partial a}\left(\begin{array}{c}
q_{1} \\
q_{2} \\
0
\end{array}\right)=\left(\begin{array}{c}
X_{11} \\
X_{21} \\
X_{31}
\end{array}\right)(1-\varepsilon)+\left(\begin{array}{c}
X_{12} \\
X_{22} \\
X_{32}
\end{array}\right)\left(-\frac{3}{2} \sqrt{\frac{1+\varepsilon}{1-\varepsilon}} M_{1}\right)
$$

To evaluate $\frac{\partial x_{i}}{\partial \varepsilon}$, we need to know $\frac{\partial q_{1}}{\partial \varepsilon}$ and $\frac{\partial q_{2}}{\partial \varepsilon}$. After differentiating the relation; $E-\varepsilon \sin E=M$, we find the following.

$$
\frac{\partial E}{\partial \varepsilon}-\sin E-\varepsilon \cos E \frac{\partial E}{\partial \varepsilon}=\frac{\partial M}{\partial \varepsilon}=0
$$

Then we find that $\frac{\partial E}{\partial \varepsilon}$ vanishes at perigee.

As results, we find the followings.

$$
\frac{\partial q_{1}}{\partial \varepsilon}=\frac{\partial}{\partial \varepsilon}[a(\cos E-\varepsilon)] \Rightarrow-a \text { and } \frac{\partial q_{2}}{\partial \varepsilon} \Rightarrow 0
$$

Accordingly, at perigee, we have

$$
\frac{\partial}{\partial \varepsilon}\left(\begin{array}{l}
x_{1} \\
x_{2} \\
x_{3}
\end{array}\right)=\left(\begin{array}{lll}
X_{11} & X_{12} & X_{13} \\
X_{21} & X_{22} & X_{23} \\
X_{31} & X_{32} & X_{33}
\end{array}\right) \frac{\partial}{\partial \varepsilon}\left(\begin{array}{c}
q_{1} \\
q_{2} \\
0
\end{array}\right)=\left(\begin{array}{c}
X_{11} \\
X_{21} \\
X_{31}
\end{array}\right)(-a)
$$

Again from the relation; $E-\varepsilon \sin E=M$, at perigee, we find the followings.

$$
\frac{\partial E}{\partial M}-\varepsilon \cos E \frac{\partial E}{\partial M}=1, \text { and } \frac{\partial E}{\partial M}=\frac{1}{1-\varepsilon}
$$

Then, at perigee, we have

$$
\begin{aligned}
& \frac{\partial q_{1}}{\partial M}=\frac{\partial}{\partial M}[a(\cos E-\varepsilon)]=-a \sin E \frac{\partial E}{\partial M} \Rightarrow 0, \text { and } \\
& \frac{\partial q_{2}}{\partial M}=\frac{\partial}{\partial M}\left[a \sqrt{1-\varepsilon^{2}} \sin E\right]=a \sqrt{1-\varepsilon^{2}} \cos E \frac{\partial E}{\partial M} \Rightarrow a \sqrt{\frac{1+\varepsilon}{1-\varepsilon}}
\end{aligned}
$$

Therefore, at perigee, we find the following.

$$
\frac{\partial}{\partial M}\left(\begin{array}{l}
x_{1} \\
x_{2} \\
x_{3}
\end{array}\right)=\left(\begin{array}{lll}
X_{11} & X_{12} & X_{13} \\
X_{21} & X_{22} & X_{23} \\
X_{31} & X_{32} & X_{33}
\end{array}\right) \frac{\partial}{\partial M}\left(\begin{array}{c}
q_{1} \\
q_{2} \\
0
\end{array}\right)=\left(\begin{array}{ccc}
X_{11} & X_{12} & X_{13} \\
X_{21} & X_{22} & X_{23} \\
X_{31} & X_{32} & X_{33}
\end{array}\right)\left(\begin{array}{c}
0 \\
a \sqrt{\frac{1+\varepsilon}{1-\varepsilon}} \\
0
\end{array}\right)=\left(\begin{array}{c}
X_{12} \\
X_{22} \\
X_{32}
\end{array}\right) a \sqrt{\frac{1+\varepsilon}{1-\varepsilon}}
$$

Evaluation of $\frac{\partial \dot{x}_{i}}{\partial s_{k}}$ at perigee can be done similarly. After then, the values of three partial derivatives $\frac{\partial \dot{x}_{i}}{\partial \Omega}, \frac{\partial \dot{x}_{i}}{\partial i}$, $\frac{\partial \dot{x}_{i}}{\partial \omega}$ and at perigee can be acquired readily as follows.

$$
\frac{\partial}{\partial \Omega}\left(\begin{array}{l}
\dot{x}_{1} \\
\dot{x}_{2} \\
\dot{x}_{3}
\end{array}\right)=\frac{\partial}{\partial \Omega}\left(\begin{array}{lll}
X_{11} & X_{12} & X_{13} \\
X_{21} & X_{22} & X_{23} \\
X_{31} & X_{32} & X_{33}
\end{array}\right)\left(\begin{array}{l}
\dot{q}_{1} \\
\dot{q}_{2} \\
\dot{q}_{3}
\end{array}\right)=\frac{\partial}{\partial \Omega}\left(\begin{array}{lll}
X_{11} & X_{12} & X_{13} \\
X_{21} & X_{22} & X_{23} \\
X_{31} & X_{32} & X_{33}
\end{array}\right) \frac{n a}{1-\varepsilon \cos E}\left(\begin{array}{c}
-\sin E \\
\sqrt{1-\varepsilon^{2}} \cos E \\
0
\end{array}\right)
$$




$$
\begin{aligned}
& \Rightarrow \frac{\partial}{\partial \Omega}\left(\begin{array}{lll}
X_{11} & X_{12} & X_{13} \\
X_{21} & X_{22} & X_{23} \\
X_{31} & X_{32} & X_{33}
\end{array}\right)\left(\begin{array}{c}
0 \\
n a \sqrt{\frac{1+\varepsilon}{1-\varepsilon}} \\
0
\end{array}\right)=\frac{\partial}{\partial \Omega}\left(\begin{array}{l}
X_{12} \\
X_{22} \\
X_{32}
\end{array}\right) n a \sqrt{\frac{1+\varepsilon}{1-\varepsilon}}
\end{aligned}
$$

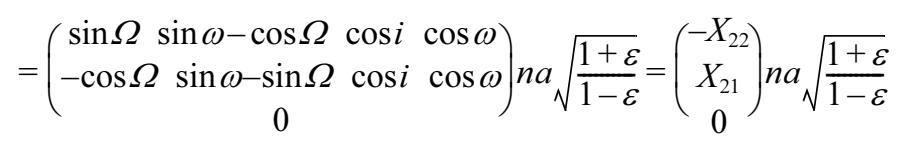

$$
\begin{aligned}
& \frac{\partial}{\partial i}\left(\begin{array}{l}
\dot{x}_{1} \\
\dot{x}_{2} \\
\dot{x}_{3}
\end{array}\right)=\frac{\partial}{\partial i}\left(\begin{array}{l}
X_{12} \\
X_{22} \\
X_{32}
\end{array}\right) n a \sqrt{\frac{1+\varepsilon}{1-\varepsilon}}=\left(\begin{array}{c}
\sin \Omega \sin i \cos \omega \\
-\cos \Omega \sin i \cos \omega \\
\cos i \cos \omega
\end{array}\right) n a \sqrt{\frac{1+\varepsilon}{1-\varepsilon}}=\left(\begin{array}{c}
X_{13} \\
X_{23} \\
X_{33}
\end{array}\right) \cos \omega n a \sqrt{\frac{1+\varepsilon}{1-\varepsilon}}
\end{aligned}
$$

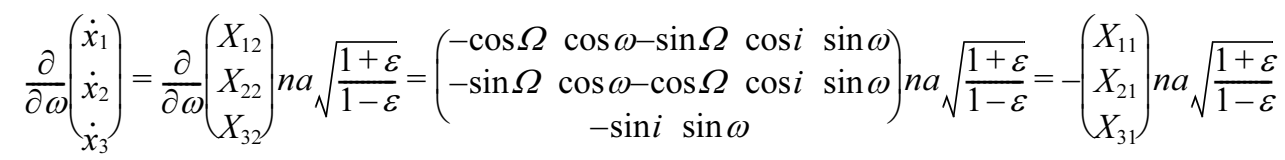

Evaluation of partial derivatives $\frac{\partial \dot{x}_{i}}{\partial a}, \frac{\partial \dot{x}_{i}}{\partial \varepsilon}$, and $\frac{\partial \dot{x}_{i}}{\partial M}$ need a little more cares. As for $\frac{\partial \dot{x}_{i}}{\partial a}$, and we can write

$$
\frac{\partial}{\partial a}\left(\begin{array}{l}
\dot{x}_{1} \\
\dot{x}_{2} \\
\dot{x}_{3}
\end{array}\right)=\left(\begin{array}{lll}
X_{11} & X_{12} & X_{13} \\
X_{21} & X_{22} & X_{23} \\
X_{31} & X_{32} & X_{33}
\end{array}\right) \frac{\partial}{\partial a}\left(\begin{array}{l}
\dot{q}_{1} \\
\dot{q}_{2} \\
\dot{q}_{3}
\end{array}\right)
$$

Then we calculate $\frac{\partial \dot{q}_{i}}{\partial a}$.

$$
\frac{\partial}{\partial a}\left(\begin{array}{c}
\dot{q}_{1} \\
\dot{q}_{2} \\
\dot{q}_{3}
\end{array}\right)=\frac{\partial}{\partial a}\left(\begin{array}{c}
n a \\
1-\varepsilon \cos E
\end{array}\left(\begin{array}{c}
-\sin E \\
\sqrt{1-\varepsilon^{2}} \cos E \\
0
\end{array}\right)\right)=\left(\frac{\partial n}{\partial a} a+n\right) \frac{1}{1-\varepsilon \cos E}\left(\begin{array}{c}
-\sin E \\
\sqrt{1-\varepsilon^{2}} \cos E \\
0
\end{array}\right)+n a \frac{\partial E}{\partial a} \frac{\partial}{\partial E}\left(\begin{array}{c}
\frac{-\sin E}{1-\varepsilon \cos E} \\
\frac{\sqrt{1-\varepsilon^{2}} \cos E}{1-\varepsilon \cos E} \\
0
\end{array}\right)
$$

$\frac{\partial n}{\partial a}$ and $\frac{\partial E}{\partial a}$ were already found above, during the derivation of Eq. (3-7d), as

$$
\frac{\partial n}{\partial a}=-\frac{3 n}{2 a} \text { and } \frac{\partial E}{\partial a}=-\frac{3 M_{1}}{2(1-\varepsilon) a}
$$

And so, at perigee $(E=0)$, we have

$$
\begin{aligned}
\frac{\partial}{\partial a}\left(\begin{array}{l}
\dot{x}_{1} \\
\dot{x}_{2} \\
\dot{x}_{3}
\end{array}\right) & =\left(\begin{array}{lll}
X_{11} & X_{12} & X_{13} \\
X_{21} & X_{22} & X_{23} \\
X_{31} & X_{32} & X_{33}
\end{array}\right) \frac{\partial}{\partial a}\left(\begin{array}{l}
\dot{q}_{1} \\
\dot{q}_{2} \\
\dot{q}_{3}
\end{array}\right)=\left(\begin{array}{lll}
X_{11} & X_{12} & X_{13} \\
X_{21} & X_{22} & X_{23} \\
X_{31} & X_{32} & X_{33}
\end{array}\right)\left(-\frac{n}{2} \sqrt{\frac{1+\varepsilon}{1-\varepsilon}}\left(\begin{array}{l}
0 \\
1 \\
0
\end{array}\right)+\frac{3 n M_{1}}{2(1-\varepsilon)^{2}}\left(\begin{array}{l}
1 \\
0 \\
0
\end{array}\right)\right) \\
& =\left(\begin{array}{l}
X_{12} \\
X_{22} \\
X_{32}
\end{array}\right)\left(-\frac{n}{2} \sqrt{\frac{1+\varepsilon}{1-\varepsilon}}\right)+\left(\begin{array}{l}
X_{11} \\
X_{21} \\
X_{31}
\end{array}\right)\left(\frac{3 n M_{1}}{2(1-\varepsilon)^{2}}\right)
\end{aligned}
$$

Evaluation of $\frac{\partial \dot{x}_{i}}{\partial \varepsilon}$ can be done as follows. 


$$
\begin{aligned}
\frac{\partial}{\partial \varepsilon}\left(\begin{array}{l}
\dot{x}_{1} \\
\dot{x}_{2} \\
\dot{x}_{3}
\end{array}\right) & \left.=\left(\begin{array}{lll}
X_{11} & X_{12} & X_{13} \\
X_{21} & X_{22} & X_{23} \\
X_{31} & X_{32} & X_{33}
\end{array}\right) \frac{\partial}{\partial \varepsilon}\left(\begin{array}{l}
\dot{q}_{1} \\
\dot{q}_{2} \\
\dot{q}_{3}
\end{array}\right)=\left(\begin{array}{ccc}
X_{11} & X_{12} & X_{13} \\
X_{21} & X_{22} & X_{23} \\
X_{31} & X_{32} & X_{33}
\end{array}\right) \frac{\partial}{\partial \varepsilon}\left(\begin{array}{c}
-\sin E \\
1-\varepsilon \cos E \\
\sqrt{1-\varepsilon^{2}} \cos E \\
0
\end{array}\right)\right) \\
& =\left(\begin{array}{lll}
X_{11} & X_{12} & X_{13} \\
X_{21} & X_{22} & X_{23} \\
X_{31} & X_{32} & X_{33}
\end{array}\right) \frac{n a \cos E}{(1-\varepsilon \sin E)^{2}}\left(\begin{array}{c}
-\sin E \\
0
\end{array}\right) \Rightarrow\left(\begin{array}{c}
X_{12} \\
X_{22} \\
X_{32}
\end{array}\right) \frac{n a}{(1-\varepsilon) \sqrt{1-\varepsilon^{2}}}+\sqrt{1-\varepsilon^{2}} \cos
\end{aligned}
$$

To evaluate $\frac{\partial \dot{x}_{i}}{\partial M}$, we need to know $\frac{\partial \dot{q}_{1}}{\partial M}$ and $\frac{\partial \dot{q}_{2}}{\partial M}$. And, at perigee, we find

$$
\begin{aligned}
& \frac{\partial \dot{q}_{1}}{\partial M}=\frac{\partial}{\partial M}\left(-n a \frac{\sin E}{1-\varepsilon \cos E}\right)=n a \frac{\partial E}{\partial M} \frac{\partial}{\partial E}\left(\frac{-\sin E}{1-\varepsilon \cos E}\right) \Rightarrow-\frac{n a}{(1-\varepsilon)^{2}}, \\
& \frac{\partial \dot{q}_{2}}{\partial M}=\frac{\partial}{\partial M}\left(n a \frac{\sqrt{1-\varepsilon^{2}} \cos E}{1-\varepsilon \cos E}\right)=n a \sqrt{1-\varepsilon^{2}} \frac{\partial E}{\partial M} \frac{\partial}{\partial E}\left(\frac{\cos E}{1-\varepsilon \cos E}\right) \Rightarrow 0 .
\end{aligned}
$$

So, at perigee, we have

$$
\frac{\partial}{\partial M}\left(\begin{array}{l}
\dot{x}_{1} \\
\dot{x}_{2} \\
\dot{x}_{3}
\end{array}\right)=\left(\begin{array}{lll}
X_{11} & X_{12} & X_{13} \\
X_{21} & X_{22} & X_{23} \\
X_{31} & X_{32} & X_{33}
\end{array}\right) \frac{\partial}{\partial M}\left(\begin{array}{l}
\dot{q}_{1} \\
\dot{q}_{2} \\
\dot{q}_{3}
\end{array}\right)=\left(\begin{array}{lll}
X_{11} & X_{12} & X_{13} \\
X_{21} & X_{22} & X_{23} \\
X_{31} & X_{32} & X_{33}
\end{array}\right)\left(\begin{array}{c}
-\frac{n a}{(1-\varepsilon)^{2}} \\
0 \\
0
\end{array}\right)=\left(\begin{array}{l}
X_{11} \\
X_{21} \\
X_{31}
\end{array}\right)\left(-\frac{n a}{(1-\varepsilon)^{2}}\right)
$$

Let us go back to the Lagrange brackets $\left[s_{l}, s_{k}\right]=\sum_{j=1}^{3}\left(\frac{\partial x_{j}}{\partial s_{l}} \frac{\partial \dot{x}_{j}}{\partial s_{k}}-\frac{\partial \dot{x}_{j}}{\partial s_{l}} \frac{\partial x_{j}}{\partial s_{k}}\right)$. We can calculate them by using Eqs. (3-7a f) and Eqs. (3-8a f). The orthonormality between column vectors of the transformation matrix $X(\Omega, i, \omega)=$ $R_{3}(-\Omega) R_{3}(-i) R_{3}(-\omega)$ has frequent application here. The Lagrange brackets are evaluated one by one as follows.

$$
\begin{aligned}
{[a, \varepsilon] } & \left.=\sum_{j=1}^{3}\left(\frac{\partial x_{j}}{\partial a} \frac{\partial \dot{x}_{j}}{\partial \varepsilon}-\frac{\partial \dot{x}_{j}}{\partial a} \frac{\partial x_{j}}{\partial \varepsilon}\right)=\left(\begin{array}{lll}
\left(X_{11}\right. & X_{21} & X_{31}
\end{array}\right)(1-\varepsilon)+\left(\begin{array}{lll}
X_{12} & X_{22} & X_{33}
\end{array}\right)\left(\begin{array}{ll}
-\frac{3}{2} \sqrt{\frac{1+\varepsilon}{1-\varepsilon}} \\
M_{1}
\end{array}\right)\right)\left(\begin{array}{l}
X_{12} \\
X_{22} \\
X_{32}
\end{array}\right) \frac{n a}{(1-\varepsilon) \sqrt{1-\varepsilon^{2}}} \\
& -\left(\begin{array}{lll}
\left(X_{12}\right. & X_{22} & X_{32}
\end{array}\right)\left(-\frac{n}{2} \sqrt{\frac{1+\varepsilon}{1-\varepsilon}}\right)+\left(\begin{array}{lll}
X_{11} & X_{21} & X_{31}
\end{array}\right)\left(\frac{3}{2} \frac{n M_{1}}{(1-\varepsilon)^{2}}\right)\left(\begin{array}{l}
X_{11} \\
X_{21} \\
X_{31}
\end{array}\right)(-a)
\end{aligned}
$$

After applying $\sum_{k=1}^{3} X_{k i} X_{k j}=\delta_{i j}$ here, we find

$$
\begin{aligned}
& {[a, \varepsilon]=-\frac{3}{2} \frac{M_{1} n a}{(1-\varepsilon)^{2}}+\frac{3}{2} \frac{n M_{1} a}{(1-\varepsilon)^{2}}=0} \\
& {[a, i]=\sum_{j=1}^{3}\left(\frac{\partial x_{j}}{\partial a} \frac{\partial \dot{x}_{j}}{\partial i}-\frac{\partial \dot{x}_{j}}{\partial a} \frac{\partial x_{j}}{\partial i}\right)=\left(\left(\begin{array}{lll}
X_{11} & X_{21} & X_{31}
\end{array}\right)(1-\varepsilon)+\left(\begin{array}{lll}
X_{12} & X_{22} & X_{32}
\end{array}\right)\left(\begin{array}{ll}
-\frac{3}{2} \sqrt{\frac{1+\varepsilon}{1-\varepsilon}} & M_{1}
\end{array}\right)\right)\left(\begin{array}{c}
X_{13} \\
X_{23} \\
X_{33}
\end{array}\right) \cos \omega n a \sqrt{\frac{1+\varepsilon}{1-\varepsilon}}} \\
& -\left(\left(\begin{array}{lll}
X_{12} & X_{22} & X_{32}
\end{array}\right)\left(-\frac{n}{2} \sqrt{\frac{1+\varepsilon}{1-\varepsilon}}\right)+\left(\begin{array}{lll}
X_{11} & X_{21} & X_{31}
\end{array}\right)\left(\frac{3}{2} \frac{n M_{1}}{(1-\varepsilon)^{2}}\right)\right)\left(\begin{array}{l}
X_{13} \\
X_{23} \\
X_{33}
\end{array}\right) \sin \omega a(1-\varepsilon)=0
\end{aligned}
$$




$$
\begin{aligned}
& {[a, \omega]=\sum_{j=1}^{3}\left(\frac{\partial x_{j}}{\partial a} \frac{\partial \dot{x}_{j}}{\partial \omega}-\frac{\partial \dot{x}_{j}}{\partial a} \frac{\partial x_{j}}{\partial \omega}\right)=\left(\left(\begin{array}{lll}
X_{11} & X_{21} & X_{31}
\end{array}\right)(1-\varepsilon)+\left(\begin{array}{lll}
X_{12} & X_{22} & X_{32}
\end{array}\right)\left(\begin{array}{ll}
-\frac{3}{2} \sqrt{\frac{1+\varepsilon}{1-\varepsilon}} & M_{1}
\end{array}\right)\right)\left(\begin{array}{l}
X_{11} \\
X_{21} \\
X_{31}
\end{array}\right)\left(\begin{array}{ll}
-n a \sqrt{\frac{1+\varepsilon}{1-\varepsilon}}
\end{array}\right)} \\
& -\left(\left(\begin{array}{lll}
X_{12} & X_{22} & X_{32}
\end{array}\right)\left(-\frac{n}{2} \sqrt{\frac{1+\varepsilon}{1-\varepsilon}}\right)+\left(\begin{array}{lll}
X_{11} & X_{21} & X_{31}
\end{array}\right)\left(\frac{3}{2} \frac{n M_{1}}{(1-\varepsilon)^{2}}\right)\right)\left(\begin{array}{l}
X_{12} \\
X_{22} \\
X_{32}
\end{array}\right) a(1-\varepsilon) \\
& =-n a \sqrt{1-\varepsilon^{2}}+\frac{n}{2} a \sqrt{1-\varepsilon^{2}}=-\frac{n a}{2} \sqrt{1-\varepsilon^{2}} \\
& {[a, \Omega]=\sum_{j=1}^{3}\left(\frac{\partial x_{j}}{\partial a} \frac{\partial \dot{x}_{j}}{\partial \Omega}-\frac{\partial \dot{x}_{j}}{\partial a} \frac{\partial x_{j}}{\partial \Omega}\right)=\left(\left(\begin{array}{lll}
X_{11} & X_{21} & X_{31}
\end{array}\right)(1-\varepsilon)+\left(\begin{array}{lll}
X_{12} & X_{22} & X_{33}
\end{array}\right)\left(\begin{array}{l}
-\frac{3}{2} \sqrt{\frac{1+\varepsilon}{1-\varepsilon}} M_{1}
\end{array}\right)\right)\left(\begin{array}{c}
-X_{22} \\
X_{12} \\
0
\end{array}\right) n a \sqrt{\frac{1+\varepsilon}{1-\varepsilon}}} \\
& -\left(\left(\begin{array}{lll}
X_{12} & X_{22} & X_{32}
\end{array}\right)\left(-\frac{n}{2} \sqrt{\frac{1+\varepsilon}{1-\varepsilon}}\right)+\left(\begin{array}{lll}
X_{11} & X_{21} & X_{31}
\end{array}\right)\left(\frac{3}{2} \frac{n M_{1}}{(1-\varepsilon)^{2}}\right)\left(\begin{array}{c}
-X_{21} \\
X_{11} \\
0
\end{array}\right) a(1-\varepsilon)\right.
\end{aligned}
$$

Here we need to know the following four coefficients.

$$
\begin{aligned}
& \left(\begin{array}{lll}
X_{11} & X_{21} & X_{31}
\end{array}\right)\left(\begin{array}{c}
-X_{22} \\
X_{12} \\
0
\end{array}\right)=-X_{11} X_{22}+X_{21} X_{12} \\
& =(\cos \Omega \cos \omega-\sin \Omega \cos i \sin \omega)(-\sin \Omega \sin \omega+\cos \Omega \cos i \cos \omega) \\
& =-\cos ^{2} \Omega \cos ^{2} \omega \cos i-\sin ^{2} \Omega \sin ^{2} \omega \cos i-\sin ^{2} \Omega \cos ^{2} \omega \cos i-\cos ^{2} \Omega \sin ^{2} \omega \cos i \\
& =-\cos ^{2} \omega \cos i-\sin ^{2} \omega \cos i=-\cos i, \\
& \left(\begin{array}{lll}
X_{12} & X_{22} & X_{32}
\end{array}\right)\left(\begin{array}{c}
-X_{22} \\
X_{12} \\
0
\end{array}\right)=-X_{12} X_{22}+X_{22} X_{12}=0,\left(\begin{array}{lll}
X_{12} & X_{22} & X_{32}
\end{array}\right)\left(\begin{array}{c}
-X_{21} \\
X_{11} \\
0
\end{array}\right)=-X_{12} X_{21}+X_{22} X_{11}=\cos i, \\
& \left(\begin{array}{lll}
X_{11} & X_{21} & X_{31}
\end{array}\right)\left(\begin{array}{c}
-X_{21} \\
X_{11} \\
0
\end{array}\right)=-X_{11} X_{21}+X_{21} X_{11}=0 \text {. }
\end{aligned}
$$

And then, we find the value of $[a, \Omega]$.

$$
[a, \Omega]=-\cos i \quad n a \sqrt{1-\varepsilon^{2}}+\cos i \frac{n}{2} a \sqrt{1-\varepsilon^{2}}=-\frac{n a}{2} \sqrt{1-\varepsilon^{2}} \cos i
$$

Next ones are found as follows.

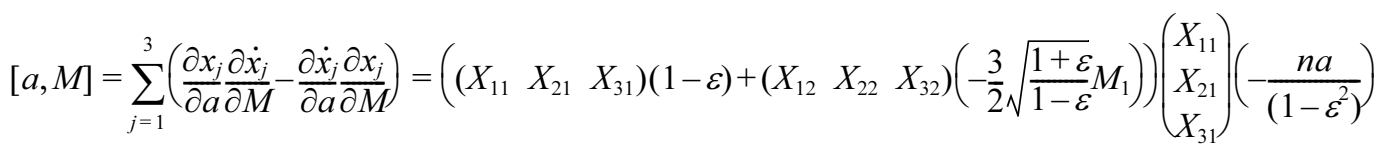

$$
\begin{aligned}
& -\left(\left(\begin{array}{lll}
X_{12} & X_{22} & X_{32}
\end{array}\right)\left(-\frac{n}{2} \sqrt{\frac{1+\varepsilon}{1-\varepsilon}}\right)+\left(\begin{array}{lll}
X_{11} & X_{21} & X_{31}
\end{array}\right)\left(\frac{3}{2} \frac{n M_{1}}{(1-\varepsilon)^{2}}\right)\right)\left(\begin{array}{l}
X_{12} \\
X_{22} \\
X_{32}
\end{array}\right) a \sqrt{\frac{1+\varepsilon}{1-\varepsilon}}=-\frac{n a}{1-\varepsilon}+\frac{n a}{2} \frac{1+\varepsilon}{1-\varepsilon}=-\frac{n a}{2}
\end{aligned}
$$

$[\varepsilon, a]=-[a, \varepsilon]=0$ 


$$
\begin{aligned}
{[\varepsilon, i] } & =\sum_{j=1}^{3}\left(\frac{\partial x_{j}}{\partial \varepsilon} \frac{\partial \dot{x}_{j}}{\partial i}-\frac{\partial \dot{x}_{j}}{\partial \varepsilon} \frac{\partial x_{j}}{\partial i}\right) \\
& =\left(\begin{array}{lll}
X_{11} & X_{21} & X_{31}
\end{array}\right)(-a)\left(\begin{array}{l}
X_{13} \\
X_{23} \\
X_{33}
\end{array}\right) \cos \omega n a \sqrt{\frac{1+\varepsilon}{1-\varepsilon}}-\left(\begin{array}{lll}
X_{12} & X_{22} & X_{32}
\end{array}\right) \frac{n a}{(1-\varepsilon) \sqrt{1-\varepsilon^{2}}}\left(\begin{array}{l}
X_{13} \\
X_{23} \\
X_{33}
\end{array}\right) \sin \omega(1-\varepsilon)
\end{aligned}
$$

Due to the orthonormality of column vectors of $X$, we find

$$
[\varepsilon, i]=0
$$

$[\varepsilon, \omega]=\sum_{j=1}^{3}\left(\frac{\partial x_{j}}{\partial \varepsilon} \frac{\partial \dot{x}_{j}}{\partial \omega}-\frac{\partial \dot{x}_{j}}{\partial \varepsilon} \frac{\partial x_{j}}{\partial \omega}\right)=\left(\begin{array}{lll}X_{11} & X_{21} & X_{31}\end{array}\right)(-a)\left(\begin{array}{c}X_{11} \\ X_{21} \\ X_{31}\end{array}\right)\left(\begin{array}{lll}-n a \sqrt{\frac{1+\varepsilon}{1-\varepsilon}}\end{array}\right)-\left(\begin{array}{lll}X_{12} & X_{22} & X_{32}\end{array}\right) \frac{n a}{(1-\varepsilon) \sqrt{1-\varepsilon^{2}}}\left(\begin{array}{c}X_{12} \\ X_{22} \\ X_{32}\end{array}\right) a(1-\varepsilon)$

$$
=n a^{2} \sqrt{\frac{1+\varepsilon}{1-\varepsilon}}-n a^{2} \frac{1}{\sqrt{1-\varepsilon^{2}}}=\frac{n a^{2} \varepsilon}{\sqrt{1-\varepsilon^{2}}}
$$

$[\varepsilon, \Omega]=\sum_{j=1}^{3}\left(\frac{\partial x_{j}}{\partial \varepsilon} \frac{\partial \dot{x}_{j}}{\partial \Omega}-\frac{\partial \dot{x}_{j}}{\partial \varepsilon} \frac{\partial x_{j}}{\partial \Omega}\right)=\left(\begin{array}{lll}X_{11} & X_{21} & X_{31}\end{array}\right)(-a)\left(\begin{array}{c}-X_{22} \\ X_{12} \\ 0\end{array}\right) n a \sqrt{\frac{1+\varepsilon}{1-\varepsilon}}-\left(\begin{array}{lll}X_{12} & X_{22} & X_{32}\end{array}\right) \frac{n a}{(1-\varepsilon) \sqrt{1-\varepsilon^{2}}}\left(\begin{array}{c}-X_{21} \\ X_{11} \\ 0\end{array}\right) a(1-\varepsilon)$

From the following equality;

$$
\left(\begin{array}{lll}
X_{11} & X_{21} & X_{31}
\end{array}\right)\left(\begin{array}{c}
-X_{22} \\
X_{12} \\
0
\end{array}\right)=-X_{11} X_{22}+X_{21} X_{12}=-\cos i=-\left(\begin{array}{lll}
X_{12} & X_{22} & X_{32}
\end{array}\right)\left(\begin{array}{c}
-X_{21} \\
X_{11} \\
0
\end{array}\right)
$$

we find

$$
[\varepsilon, \Omega]=n a^{2} \sqrt{\frac{1+\varepsilon}{1-\varepsilon}} \cos i-n a^{2} \frac{1}{\sqrt{1-\varepsilon^{2}}} \cos i=\frac{n a^{2}}{\sqrt{1-\varepsilon^{2}}} \cos i
$$

And then we find the followings.

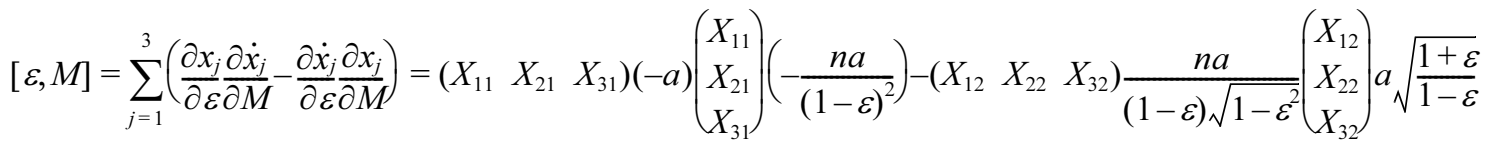

$$
\begin{aligned}
& =\frac{n a^{2}}{(1-\varepsilon)^{2}}-\frac{n a^{2}}{(1-\varepsilon)^{2}}=0 \\
& {[i, a]=-[a, i]=0,[i, \varepsilon]=-[\varepsilon, i]=0} \\
& {[i, \omega]=\sum_{j=1}^{3}\left(\frac{\partial x_{j}}{\partial i} \frac{\dot{x}_{j}}{\partial \omega}-\frac{\partial \dot{x}_{j}}{\partial i} \frac{\partial x_{j}}{\partial \omega}\right)} \\
& =\left(\begin{array}{llll}
X_{13} & X_{23} & X_{33}
\end{array}\right) \sin \omega a(1-\varepsilon)\left(\begin{array}{l}
X_{11} \\
X_{21} \\
X_{31}
\end{array}\right)\left(\begin{array}{lll}
-n a \sqrt{\frac{1+\varepsilon}{1-\varepsilon}}
\end{array}\right)-\left(\begin{array}{lll}
X_{13} & X_{23} & X_{33}
\end{array}\right) \cos \omega n a \sqrt{\frac{1+\varepsilon}{1-\varepsilon}}\left(\begin{array}{c}
X_{12} \\
X_{22} \\
X_{32}
\end{array}\right) a(1-\varepsilon)=0
\end{aligned}
$$




$$
\begin{aligned}
{[i, \Omega] } & =\sum_{j=1}^{3}\left(\frac{\partial x_{j}}{\partial i} \frac{\partial \dot{x}_{j}}{\partial \Omega}-\frac{\partial \dot{x}_{j}}{\partial i} \frac{\partial x_{j}}{\partial \Omega}\right) \\
& =\left(\begin{array}{lllll}
X_{13} & X_{23} & X_{33}
\end{array}\right) \sin \omega a(1-\varepsilon)\left(\begin{array}{c}
-X_{22} \\
X_{12} \\
0
\end{array}\right) n a \sqrt{\frac{1+\varepsilon}{1-\varepsilon}}-\left(\begin{array}{lll}
X_{13} & X_{23} & X_{33}
\end{array}\right) \cos \omega n a \sqrt{\frac{1+\varepsilon}{1-\varepsilon}}\left(\begin{array}{c}
-X_{21} \\
X_{11} \\
0
\end{array}\right) a(1-\varepsilon)
\end{aligned}
$$

Using the two equalities;

$$
\left(\begin{array}{lll}
X_{13} & X_{23} & X_{33}
\end{array}\right)\left(\begin{array}{c}
-X_{22} \\
X_{12} \\
0
\end{array}\right)=\sin i \sin \omega \text { and }\left(\begin{array}{lll}
X_{13} & X_{23} & X_{33}
\end{array}\right)\left(\begin{array}{c}
-X_{21} \\
X_{11} \\
0
\end{array}\right)=-\sin i \cos \omega,
$$

we find

$$
[i, \Omega]=n a^{2} \sqrt{1-\varepsilon^{2}} \sin i
$$

Next Lagrange brackets are acquired as followings.

$$
\begin{aligned}
& {[i, M]=\sum_{j=1}^{3}\left(\frac{\partial x_{j}}{\partial i} \frac{\partial \dot{x}_{j}}{\partial M}-\frac{\partial \dot{x}_{j}}{\partial i} \frac{\partial x_{j}}{\partial M}\right)} \\
& =\left(\begin{array}{lll}
X_{13} & X_{23} & X_{33}
\end{array}\right) \sin \omega a(1-\varepsilon)\left(\begin{array}{l}
X_{11} \\
X_{21} \\
X_{31}
\end{array}\right)\left(\begin{array}{lll}
-\frac{n a}{(1-\varepsilon)^{2}}
\end{array}\right)-\left(\begin{array}{lll}
X_{13} & X_{23} & X_{33}
\end{array}\right) \cos \omega n a \sqrt{\frac{1+\varepsilon}{1-\varepsilon}}\left(\begin{array}{l}
X_{12} \\
X_{22} \\
X_{32}
\end{array}\right) a \sqrt{\frac{1+\varepsilon}{1-\varepsilon}}=0 \\
& {[\omega, a]=-[a, \omega]=\frac{n a}{2} \sqrt{1-\varepsilon^{2}},[\omega, \varepsilon]=-[\varepsilon, \omega]=-\frac{n a^{2} \varepsilon}{\sqrt{1-\varepsilon^{2}}},[\omega, i]=-[i, \omega]=0} \\
& {[\omega, \Omega]=\sum_{j=1}^{3}\left(\frac{\partial x_{j}}{\partial \omega} \frac{\partial \dot{x}_{j}}{\partial \Omega}-\frac{\partial \dot{x}_{j}}{\partial \omega} \frac{\partial x_{j}}{\partial \Omega}\right)} \\
& =\left(\begin{array}{lll}
X_{12} & X_{22} & X_{32}
\end{array}\right) a(1-\varepsilon)\left(\begin{array}{c}
-X_{22} \\
X_{12} \\
0
\end{array}\right)\left(\begin{array}{lll}
-n a \sqrt{\frac{1+\varepsilon}{1-\varepsilon}}
\end{array}\right)-\left(\begin{array}{lll}
X_{13} & X_{23} & X_{33}
\end{array}\right)\left(\begin{array}{l}
-n a \sqrt{\frac{1+\varepsilon}{1-\varepsilon}}
\end{array}\right)\left(\begin{array}{c}
-X_{21} \\
X_{11} \\
0
\end{array}\right) a(1-\varepsilon)=0 \\
& {[\omega, M]=\sum_{j=1}^{3}\left(\frac{\partial x_{j}}{\partial \omega} \frac{\partial \dot{x}_{j}}{\partial M}-\frac{\partial \dot{x}_{j}}{\partial \omega} \frac{\partial x_{j}}{\partial M}\right)} \\
& =\left(\begin{array}{lll}
X_{12} & X_{22} & X_{32}
\end{array}\right) a(1-\varepsilon)\left(\begin{array}{l}
X_{11} \\
X_{21} \\
X_{31}
\end{array}\right)\left(\begin{array}{ll}
-\frac{n a}{(1-\varepsilon)^{2}}
\end{array}\right)-\left(\begin{array}{lll}
X_{11} & X_{21} & X_{31}
\end{array}\right)\left(-n a \sqrt{\frac{1+\varepsilon}{1-\varepsilon}}\right)\left(\begin{array}{l}
X_{12} \\
X_{22} \\
X_{32}
\end{array}\right) \sqrt{\frac{1+\varepsilon}{1-\varepsilon}}=0 \\
& {[\Omega, a]=-[a, \Omega]=\frac{n a}{2} \sqrt{1-\varepsilon^{2}} \cos i,[\Omega, \varepsilon]=-[\varepsilon, \Omega]=-\frac{n a^{2} \varepsilon}{\sqrt{1-\varepsilon^{2}}} \cos i} \\
& {[\Omega, i]=-[i, \Omega]=-n a^{2} \sqrt{1-\varepsilon^{2}} \sin i,[\Omega, \omega]=-[\omega, \Omega]=0} \\
& {[\Omega, M]=\sum_{j=1}^{3}\left(\frac{\partial x_{j}}{\partial \Omega} \frac{\partial \dot{x}_{j}}{\partial M}-\frac{\partial \dot{x}_{j}}{\partial \Omega} \frac{\partial x_{j}}{\partial M}\right)} \\
& =\left(\begin{array}{lll}
-X_{21} & X_{11} & 0
\end{array}\right) a(1-\varepsilon)\left(\begin{array}{c}
X_{11} \\
X_{21} \\
X_{31}
\end{array}\right)\left(\begin{array}{ccc}
-\frac{n a}{(1-\varepsilon)^{2}}
\end{array}\right)-\left(\begin{array}{lll}
-X_{22} & X_{12} & 0
\end{array}\right) n a \sqrt{\frac{1+\varepsilon}{1-\varepsilon}}\left(\begin{array}{c}
X_{12} \\
X_{22} \\
X_{32}
\end{array}\right) \sqrt{\frac{1+\varepsilon}{1-\varepsilon}}=0
\end{aligned}
$$


We find the values of Lagrange brackets associated with mean anomaly as follows.

$$
\begin{aligned}
& {[M, a]=-[a, M]=\frac{n a}{2},} \\
& {[M, \varepsilon]=-[\varepsilon, M]=0,[M, i]=-[i, M]=0,[M, \omega]=-[\omega, M]=0,[M, \Omega]=-[\Omega, M]=0}
\end{aligned}
$$

We have evaluated all the values of the Lagrange brackets, only twelve of which are nonzero. Actually only half of the twelve are independent (antisymmetry).

If we write the Eq. (3-3) in matrix form, we have the following.

$$
\left(\begin{array}{lc}
{[a, a][a, \varepsilon][a, i]} & {[a, \omega][a, \Omega][a, M]} \\
{[\varepsilon, a][\varepsilon, \varepsilon][\varepsilon, i]} & {[\varepsilon, \omega][\varepsilon, \Omega][\varepsilon, M]} \\
{[i, a][i, \varepsilon][i, i]} & {[i, \omega][i, \Omega][i, M]} \\
{[\omega, a][\omega, \varepsilon][\omega, i]} & {[\omega, \omega][\omega, \Omega][\omega, M]} \\
{[\Omega, a][\Omega, \varepsilon][\Omega, i]} & {[\Omega, \omega][\Omega, \Omega][\Omega, M]} \\
{[M, a][M, \varepsilon][M, i]} & {[M, \omega][M, \Omega][M, M]}
\end{array}\right)\left(\begin{array}{l}
d a / d t \\
d \varepsilon / d t \\
d i / d t \\
d \omega / d t \\
d \Omega / d t \\
d M / d t
\end{array}\right)=-\left(\begin{array}{l}
\partial F / \partial a \\
\partial F / \partial \varepsilon \\
\partial F / \partial i \\
\partial F / \partial \omega \\
\partial F / \partial \Omega \\
\partial F / \partial M
\end{array}\right)
$$

This equation can now be explicitly shown as the followings.

$$
\begin{aligned}
& -\frac{n a}{2}\left(\sqrt{1-\varepsilon^{2}} \frac{d \omega}{d t}+\sqrt{1-\varepsilon^{2}} \cos i \frac{d \Omega}{d t}+\frac{d M}{d t}\right)=-\frac{\partial F}{\partial a}, \\
& \frac{n a^{2} \varepsilon}{\sqrt{1-\varepsilon^{2}}}\left(\frac{d \omega}{d t}+\cos i \frac{d \Omega}{d t}\right)=-\frac{\partial F}{\partial \varepsilon}, \\
& n a^{2} \sqrt{1-\varepsilon^{2}} \sin i \frac{d \Omega}{d t}=-\frac{\partial F}{\partial i} \\
& \frac{n a}{2} \sqrt{1-\varepsilon^{2}} \frac{d a}{d t}-\frac{n a^{2} \varepsilon}{\sqrt{1-\varepsilon^{2}}} \frac{d \varepsilon}{d t}=-\frac{\partial F}{\partial \omega} \\
& \frac{n a}{2} \sqrt{1-\varepsilon^{2}} \cos i \frac{d a}{d t}-\frac{n a^{2} \varepsilon}{\sqrt{1-\varepsilon^{2}}} \cos i \frac{d \varepsilon}{d t}-n a^{2} \sqrt{1-\varepsilon^{2}} \sin i \frac{d i}{d t}=-\frac{\partial F}{\partial \Omega} \\
& \frac{n a}{2} \frac{d a}{d t}=-\frac{\partial F}{\partial M}
\end{aligned}
$$

This set of six Eqs. (3-10a $\sim$ ) can be rewritten in the form of $\frac{d s_{j}}{d t}=\sum_{k=1}^{6} A_{j k} \frac{\partial F}{\partial s_{k}}$ as follows. First, we directly have the expressions of $\frac{d a}{d t}$ and $\frac{d \Omega}{d t}$ from Eqs (3-10c, f).

$$
\frac{d \Omega}{d t}=-\frac{1}{n a^{2} \sqrt{1-\varepsilon^{2}} \sin i} \frac{\partial F}{\partial i} \text { and } \frac{d a}{d t}=-\frac{2}{n a} \frac{\partial F}{\partial M}
$$

From Eq. (3-10d), we find

$$
\frac{n a^{2} \varepsilon}{\sqrt{1-\varepsilon^{2}}} \frac{d \varepsilon}{d t}=\frac{\partial F}{\partial \omega}+\frac{n a}{2} \sqrt{1-\varepsilon^{2}} \frac{d a}{d t}
$$

And then, we have

$$
\frac{d \varepsilon}{d t}=\frac{\sqrt{1-\varepsilon^{2}}}{n a^{2} \varepsilon}\left(\frac{\partial F}{\partial \omega}-\sqrt{1-\varepsilon^{2}} \frac{\partial F}{\partial M}\right)
$$


From Eq. (3-10b), we have

$$
\frac{d \omega}{d t}=-\cos i \frac{d \Omega}{d t}-\frac{\sqrt{1-\varepsilon^{2}}}{n a^{2} \varepsilon} \frac{\partial F}{\partial \varepsilon}=\frac{\cos i}{n a^{2} \sqrt{1-\varepsilon^{2}} \sin i} \frac{\partial F}{\partial i}-\frac{\sqrt{1-\varepsilon^{2}}}{n a^{2} \varepsilon} \frac{\partial F}{\partial \varepsilon}
$$

From Eq. (3-10e), we have

$$
\frac{d i}{d t}=\frac{1}{n a^{2} \sqrt{1-\varepsilon^{2}} \sin i} \frac{\partial F}{\partial \Omega}+\frac{\cos i}{2 a \sin i} \frac{d a}{d t}-\frac{\varepsilon \cos i}{\left(1-\varepsilon^{2}\right) \sin i} \frac{d \varepsilon}{d t}=\frac{1}{n a^{2} \sqrt{1-\varepsilon^{2}} \sin i} \frac{\partial F}{\partial \Omega}-\frac{\cos i}{n a^{2} \sqrt{1-\varepsilon^{2}} \sin i} \frac{\partial F}{\partial \omega}
$$

From Eq. (3-10a), we have

$$
\frac{d M}{d t}=\frac{2}{n a} \frac{\partial F}{\partial a}-\sqrt{1-\varepsilon^{2}} \cos i \frac{d \Omega}{d t}-\sqrt{1-\varepsilon^{2}} \frac{d \omega}{d t}=\frac{2}{n a} \frac{\partial F}{\partial a}+\frac{\left(1-\varepsilon^{2}\right)}{n a^{2} \varepsilon} \frac{\partial F}{\partial \varepsilon}
$$

Rearranging these six expressions for $\frac{d s_{j}}{d t}$, we find

$$
\begin{aligned}
& \frac{d a}{d t}=-\frac{2}{n a} \frac{\partial F}{\partial M}, \\
& \frac{d \varepsilon}{d t}=\frac{\sqrt{1-\varepsilon^{2}}}{n a^{2} \varepsilon}\left(\frac{\partial F}{\partial \omega}-\sqrt{1-\varepsilon^{2}} \frac{\partial F}{\partial M}\right), \\
& \frac{d i}{d t}=\frac{1}{n a^{2} \sqrt{1-\varepsilon^{2}} \sin i}\left(\frac{\partial F}{\partial \Omega}-\cos i \frac{\partial F}{\partial \omega}\right), \\
& \frac{d \omega}{d t}=\frac{1}{n a^{2}}\left(\frac{\cos i}{\sqrt{1-\varepsilon^{2}} \sin i} \frac{\partial F}{\partial i}-\frac{\sqrt{1-\varepsilon^{2}}}{\varepsilon} \frac{\partial F}{\partial \varepsilon}\right), \\
& \frac{d \Omega}{d t}=-\frac{1}{n a^{2} \sqrt{1-\varepsilon^{2}} \sin i} \frac{\partial F}{\partial i}, \\
& \frac{d M}{d t}=\frac{2}{n a} \frac{\partial F}{\partial a}+\frac{\left(1-\varepsilon^{2}\right)}{n a^{2} \varepsilon} \frac{\partial F}{\partial \varepsilon}
\end{aligned}
$$

As described in the former section, shape of Keplerian elliptical orbit is not changing in time under central potential $V_{\text {central }}=-\frac{G M_{e}}{r}$. In fact, while the satellite moves along the elliptical orbit, its mean anomaly $M$ increases linearly with time; $d M=n d t$. We define $R$ as the deviation of the potential from central potential, then

$$
R=V-V_{\text {central }}=V+\frac{G M_{e}}{r}
$$

Finally the perturbed equation of motion for satellite, Eq. (3-11), can be rewritten in terms of $\frac{\partial R}{\partial s_{k}}$ without any difference in mathematical expression except the sixth one for $\frac{d M}{d t}$; Eq (3-11f). Since $F=T+V$, $\frac{\partial F}{\partial a}$ is related with as follows.

$$
\frac{\partial F}{\partial a}=\frac{\partial}{\partial a}(T+V)=\frac{\partial}{\partial a}\left(T-\frac{G M_{e}}{r}+R\right)
$$

According to Eq. (2-26), we have $\frac{\partial}{\partial a}\left(T-\frac{G M_{e}}{r}\right)=\frac{\partial}{\partial a}\left(-\frac{G M_{e}}{2 a}\right)=\frac{G M_{e}}{2 a^{2}}$, and also, from the definition of $n$ in the 
former section, we have $\frac{G M_{e}}{a^{3}}=n^{2}$. Therefore we can rewrite the right hand side of Eq. (3-11f) as follows.

$$
\frac{d M}{d t}=\frac{2}{n a} \frac{\partial F}{\partial a}+\frac{\left(1-\varepsilon^{2}\right)}{n a^{2} \varepsilon} \frac{\partial F}{\partial \varepsilon}=\frac{2}{n a}\left(\frac{\partial R}{\partial a}+\frac{G M_{e}}{2 a^{2}}\right)+\frac{\left(1-\varepsilon^{2}\right)}{n a^{2} \varepsilon} \frac{\partial F}{\partial \varepsilon}=\frac{2}{n a} \frac{\partial R}{\partial a}+n+\frac{\left(1-\varepsilon^{2}\right)}{n a^{2} \varepsilon} \frac{\partial R}{\partial \varepsilon}
$$

Finally we state the equation of motion for satellite under perturbing potential $R$ as

$$
\begin{aligned}
& \frac{d a}{d t}=-\frac{2}{n a} \frac{\partial R}{\partial M}, \\
& \frac{d \varepsilon}{d t}=\frac{\sqrt{1-\varepsilon^{2}}}{n a^{2} \varepsilon}\left(\frac{\partial R}{\partial \omega}-\sqrt{1-\varepsilon^{2}} \frac{\partial R}{\partial M}\right), \\
& \frac{d i}{d t}=\frac{1}{n a^{2} \sqrt{1-\varepsilon^{2}} \sin i}\left(\frac{\partial R}{\partial \Omega}-\cos i \frac{\partial R}{\partial \omega}\right), \\
& \frac{d \omega}{d t}=\frac{1}{n a^{2}}\left(\frac{\cos i}{\sqrt{1-\varepsilon^{2}} \sin i} \frac{\partial R}{\partial i}-\frac{\sqrt{1-\varepsilon^{2}}}{\varepsilon} \frac{\partial R}{\partial \varepsilon}\right), \\
& \frac{d \Omega}{d t}=-\frac{1}{n a^{2} \sqrt{1-\varepsilon^{2}} \sin i} \frac{\partial R}{\partial i}, \\
& \frac{d M}{d t}=n+\frac{2}{n a} \frac{\partial R}{\partial a}+\frac{\left(1-\varepsilon^{2}\right)}{n a^{2} \varepsilon} \frac{\partial R}{\partial \varepsilon}
\end{aligned}
$$

\section{Conclusion}

We provided full derivation of the Kaula's formulation of the perturbed equation of motion for satellites under noncentral potential. Also we provided set of proofs of the Kepler's laws for planet/satellite. In Appendix, we described two different ways to deduce Keplerian elliptical orbit and others including discussion about Lagrange bracket and Poisson bracket.

\section{Acknowledgements}

This work was supported by KASI (KASI=Korea Astronomy and Space Science Institute) as one of its 'Basic Core Technology Development' programs. Yumin Jean carefully followed the content of this article and checked its validity. Sung-Ho $\mathrm{Na}$ appreciates his early discipline in the Ohio State University and helping suggestions from JKESS editorial board.

\section{References}

Brouwer, D. and Clemence G. M., 1961, Methods of Celestial Mechanics, Academic Press.

Danby, J. M. A., 1962, Celestial Mechanics, The Macmillian Company.

Gradshteyn, I. S. and Ryzhik, I. M., 1965, Table of Integrals, Series, and Products, Academic Press, Inc., 2.261 .

Kaula, W. M., 1966, Theory of Satellite Geodesy, Blaisdell Publishing Company. (Dover reprint available)

Pollard, H, 1966, Mathematical Introduction to Celestial Mechanics, Prentice-Hall Inc.

Seeber, G., 2003, Satellite Geodesy 2nd edition, Walter de Gruyter.

Sterne, T. E., 1960, An Introduction to Celestial Mechanics, Interscience Publishers, Inc.

2012년 1월 4일 접수 2012년 2월 3일 수정원고 접수 2012년 2월 22일 채택 


\section{Appendix 1. Alternative derivations of Kepler's 1st law}

The Kepler's 2nd and 3rd laws can be deduced from the Newton's law of gravitation in a straightforward manner. But derivation of the Keplerian ellipse $r=r(\phi)$ as in Eq. (2-15a) through direct integration is not quite obvious.

Following is a slight abridgement of the derivation of the Kepler's 1st law supported by Seeber (Seeber, 2003). Similar treatment can be found in Pollard (Pollard, 1966). Newton's equation of motion for satellite, Eq. (2-4) is again stated as

$$
\stackrel{\vec{r}}{\mu}=-G m_{1} m_{2} \frac{\vec{r}}{r^{3}} \Rightarrow \stackrel{\vec{r}}{r}=-G\left(m_{1}+m_{2}\right) \frac{\vec{r}}{r^{3}}
$$

Define a vector $\vec{h}$ as $\vec{h}=\vec{r} \times \vec{v}$, then, by taking cross product to Eq. (A1-1) and $\vec{h}$, we have

$$
\stackrel{\vec{r}}{r} \times \vec{h}=\vec{h} \times \vec{r} G\left(m_{1}+m_{2}\right) r^{-3}
$$

Since $\vec{h}$ is constant in time (proportional to the angular momentum $\vec{l}$ ), the left hand side of Eq. (A1-2) can be rewritten as $\frac{d}{d t}(\vec{v} \times \vec{h})$. The right hand side of Eq. (A1-2) can be rewritten as

$$
\begin{aligned}
& \vec{h} \times \vec{r} G\left(m_{1}+m_{2}\right) r^{-3}=[(\vec{r} \times \vec{v}) \times \vec{r}] G\left(m_{1}+m_{2}\right) r^{-3} \\
& =\left[r^{2} \vec{v}-(\vec{r} \bullet \vec{v}) \vec{r}\right] G\left(m_{1}+m_{2}\right) r^{-3} \\
& =G\left(m_{1}+m_{2}\right)\left(r^{-1} \vec{v}-r^{-2} \overrightarrow{r r}\right) \\
& =G\left(m_{1}+m_{2}\right) \frac{d}{d t}\left(\frac{\vec{r}}{r}\right),
\end{aligned}
$$

For the 3rd step of Eq. (A1-3), we used the following.

$$
\vec{r} \bullet \vec{v}=\vec{r} \bullet(\dot{r} \hat{r}+r \dot{\phi} \hat{\phi})=r \dot{r}
$$

Then Eq. (A1-2) can be written as

$$
\frac{d}{d t}(\vec{v} \times \vec{h})=G\left(m_{1}+m_{2}\right) \frac{d}{d t}\left(\frac{\vec{r}}{r}\right)
$$

Integration of Eq. (A1-4) leads to

$$
\vec{v} \times \vec{h}=G\left(m_{1}+m_{2}\right) \frac{\vec{r}}{r}+\vec{B}
$$

where $\vec{B}$ is the constant vector of integration and is confined to be on the orbital plane, because $\vec{B}$ must be a linear combination of $\vec{v} \times \vec{h}$ and $\vec{r}$. Taking inner product of Eq. (A1-5) with $\vec{r}$ leads to

$$
\vec{r} \bullet(\vec{v} \times \vec{h})=\vec{h} \bullet(\vec{r} \times \vec{v})=h^{2}=G\left(m_{1}+m_{2}\right) r+\vec{r} \bullet \vec{B}
$$


which is rewritten as the following relation.

$$
r=\frac{h^{2} /\left(G\left(m_{1}+m_{2}\right)\right)}{1+\frac{B}{G\left(m_{1}+m_{2}\right)} \cos \phi}=\frac{p}{1+\varepsilon \cos \phi},
$$

where $p$ and $\varepsilon$ are defined as follows.

$p=\frac{h^{2}}{G\left(m_{1}+m_{2}\right)}=a\left(1-\varepsilon^{2}\right), \quad \varepsilon=\frac{B}{G\left(m_{1}+m_{2}\right)}$

Two former equations of Kepler's elliptical orbit; Eq. (2-15) and Eq. (2-20) are equivalent to Eq. (A1-6a).

Another derivation is possible from the equation of motion; Eq. (A1-1). First, the time derivatives $\vec{r}$ and $\vec{r}$ are acquired as follows.

$$
\begin{aligned}
& \stackrel{\vec{r}}{r}=\frac{d}{d t}(r \hat{r})=\dot{r} \hat{r}+r \dot{\phi} \hat{\phi} \\
& \stackrel{\vec{r}}{=} \frac{d}{d t} \dot{\vec{r}}=\left(\ddot{r}-r \dot{\phi}^{2}\right) \hat{r}+(2 \dot{r} \dot{\phi}+r \ddot{\phi}) \hat{\phi}
\end{aligned}
$$

Combining Eq. (A1-7b) with Eq. (A1-1), we have the following equality.

$$
\ddot{r}-r \dot{\phi}^{2}=-G \frac{\left(m_{1}+m_{2}\right)}{r^{2}}
$$

As before, we define the variable $u$ as $u=\frac{1}{r}$. Then we find expressions for $\dot{r}$ and $\ddot{r}$ in terms of $u$ and its derivatives; $\frac{d u}{d \phi}$ and $\frac{d^{2} u}{d \phi^{2}}$, as follows.

$$
\dot{r}=-\frac{1}{u^{2}} \dot{u}=-\frac{1}{u^{2}} \frac{d u}{d \phi} \dot{\phi}=-\frac{l}{\mu} \frac{d u}{d \phi}
$$

where angular momentum $l$ is defined $l=\mu r^{2} \dot{\phi}$, as before. Similarly we find

$$
\ddot{r}=-\frac{l^{2}}{\mu^{2}} u^{2} \frac{d^{2} u}{d \phi^{2}}
$$

Combining (A1-8) and (A1-9), we find the following equation.

$$
\frac{d^{2} u}{d \phi^{2}}+u=\frac{\mu^{2}}{l^{2}} G\left(m_{1}+m_{2}\right)
$$

The solution for Eq. (A1-10) follows directly as

$$
u=\frac{1}{r}=\frac{\mu^{2}}{l^{2}} G\left(m_{1}+m_{2}\right)+A \cos \left(\phi-\phi_{0}\right)
$$

Eq. (A1-11) is equivalent to Eq. (2-15) or other expressions for Keplerian ellipse. This derivation has been favored in textbooks. 


\section{Appendix 2. Sterne's derivation and the Relation between Poisson bracket and Lagrange bracket}

In the beginning part of main section, we attained the equation of motion for satellite as,

$$
\sum_{k=1}^{6}\left[s_{l}, s_{k}\right] \frac{d s_{k}}{d t}=-\frac{\partial F}{\partial s_{l}}
$$

where $\left[s_{l}, s_{k}\right]$ is the Lagrange bracket. The remaining part of main section was to find $\frac{d s_{k}}{d t}$ by using Lagrange brackets and other manipulations.

In fact, we can do this differently by using Poisson bracket $\left(s_{l}, s_{k}\right)$ as Sterne did (Sterne, 1960). Suppose we know a set of canonical variables $q_{i}$ and $p_{i}$ for satellite. Then we can express the time derivative of Keplerian orbital element $s_{k}$ as,

$$
\frac{d s_{k}}{d t}=\sum_{i=1}^{3}\left(\frac{\partial s_{k}}{\partial q_{i}} \dot{q}_{i}+\frac{\partial s_{k}}{\partial p_{i}} \dot{p}_{i}\right)
$$

Canonical variables have the following properties.

$$
\dot{q}_{i}=\frac{\partial H}{\partial p_{i}}, \dot{p}_{i}=-\frac{\partial H}{\partial q_{i}}
$$

Then we have follows.

$$
\frac{d s_{k}}{d t}=\sum_{i=1}^{3}\left(\frac{\partial s_{k}}{\partial q_{i}} \frac{\partial H}{\partial p_{i}}-\frac{\partial s_{k}}{\partial p_{i}} \frac{\partial H}{\partial q_{i}}\right)=\sum_{i=1}^{3}\left(\frac{\partial s_{k}}{\partial q_{i}} \sum_{l=1}^{6} \frac{\partial H}{\partial s_{l}} \frac{\partial s_{l}}{\partial p_{i}}-\frac{\partial s_{k}}{\partial p_{i}} \sum_{l=1}^{6} \frac{\partial H}{\partial s_{l}} \frac{\partial s_{l}}{\partial q_{i}}\right)=\sum_{l=1}^{6} \sum_{i=1}^{3}\left(\frac{\partial s_{k}}{\partial q_{i}} \frac{\partial s_{l}}{\partial p_{i}}-\frac{\partial s_{k}}{\partial p_{i}} \frac{\partial s_{l}}{\partial q_{i}}\right) \frac{\partial H}{\partial s_{l}}
$$

The cartesian coordinates and associated linear momenta compose a set of canonical variables. Hamiltonian $H$ is identified as the total energy $F=T+V$. Then we can rewrite the time derivative of Keplerian element $\frac{\partial s_{k}}{d t}$ as follows.

$$
\frac{d s_{k}}{d t}=\sum_{l=1}^{6}\left(s_{k}, s_{l}\right) \frac{\partial F}{\partial s_{l}}
$$

where the Poisson bracket $\left(s_{k}, s_{l}\right)$ is defined as

$$
\left(s_{k}, s_{l}\right)=\sum_{i=1}^{3}\left(\frac{\partial s_{k}}{\partial x_{i}} \frac{\partial s_{l}}{\partial \dot{x}_{i}}-\frac{\partial s_{k}}{\partial \dot{x}_{i}} \frac{\partial s_{l}}{\partial x_{i}}\right)
$$

We once again write the definition of Lagrange bracket.

$$
\left[s_{k}, s_{l}\right]=\sum_{i=1}^{3}\left(\frac{\partial x_{i}}{\partial s_{k}} \frac{\partial \dot{x}_{i}}{\partial s_{l}}-\frac{\partial \dot{x}_{i}}{\partial s_{k}} \frac{\partial x_{i}}{\partial s_{l}}\right)
$$

From comparison of the two equivalent equations of motion; Eqs. (A2-1) and (A2-4), we notice the inverse relation between the Lagrange bracket and the Poisson bracket. Two matrices composed of each kinds of bracket are negative of inverse of each other. It is noted that evaluation of Poisson bracket requires explicit form of $s_{k}$ in terms of $x_{i}$ and $\dot{x}_{i}$; that is $s_{k}\left(x_{i}, \dot{x}_{i}\right)$. 